\title{
QUASI-STATIC EVOLUTION FOR FATIGUE DEBONDING
}

\author{
Alessandro FERriero ${ }^{1}$
}

\begin{abstract}
The propagation of fractures in a solid undergoing cyclic loadings is known as the fatigue phenomenon. In this paper, we present a time continuous model for fatigue, in the special situation of the debonding of thin layers, coming from a time discretized version recently proposed by Jaubert and Marigo [C. R. Mecanique 333 (2005) 550-556]. Under very general assumptions on the surface energy density and on the applied displacement, we discuss the well-posedness of our problem and we give the main properties of the evolution process.
\end{abstract}

Mathematics Subject Classification. 74R10, 49M99, 49J40

Received December 14, 2005. Revised October 8, 2006.

Published online September 21, 2007.

\section{INTRODUCTION}

In 1998 [7], Francfort and Marigo proposed a variational theory of brittle fracture which does away with some important defects of the classical Griffith theory [10], such as the impossibility of crack initiation, the a priori knowledge of the crack path and the the high regularity of the crack zone.

The main idea, borrowed from Mumford-Shah model for image segmentation [12], is that the crack wants to quasi-statically minimize its total energy among all competitors. In other words, at any time the crack must minimize the elastic energy of the uncracked part of the material plus the surface energy of the crack among all possible cracks greater than the previous one (cracks cannot disappear). Furthermore, to recover the propagation criteria of Griffith theory in the current setting, the evolution is also constrained to satisfy an energy balance between the work of the external forces and the mechanical energy of the system.

Following Griffith's hypothesis, the surface energy of the crack is proportional to the surface area of the crack, independently of the value of the displacement jump. Because of that, the model proposed in [7] cannot provide crack propagation for fatigue.

We recall briefly that the fatigue phenomenon is the growth of cracks in a structure submitted to cyclic loadings. It can be observed that fractures propagate with each cycle even if the maximal intensity of the applied loads remain constant.

In a recent work [11], Jaubert and Marigo have extended the variational model of fracture evolution to also provide for fatigue. As a first approach, they consider the problem of the debonding of a thin layer initially glued to a fixed substrate and submitted to a cyclic deflection at one tip (that can be seen as a simplified two-dimensional crack evolution model).

Keywords and phrases. Variational models, quasistatic evolution, rate-independent processes, fatigue, fractures.

1 CMAP, École Polytechnique, 91128 Palaiseau, France; ferriero@cmapx.polytechnique.fr 
The new ingredients introduced in [11] are a memory field of the displacement history and a surface energy density depending on the displacement jump, instead of the Griffith energy. The model is therefore written in terms of a family of time discrete evolutions. At each time step, a static variational problem is solved, and time evolution is considered through an irreversibility condition on the memory field.

In this paper, we formulate a time continuous evolution model for the process presented in [11], in the spirit of [3-8], and we prove an existence result for such an evolution. In [8] and references therein, the physical meaning of those formulations is discussed.

The continuous model is based on two equations: the energy balance, that is the mechanical version of the second law of thermodynamics, and the stability condition, that is the minimality property postulated for the evolution at any fixed time.

Two difficult tasks are faced in order to obtain the continuous formulation. The surface energy density is concave and bounded. That complicates the analysis since we look for a priori estimates. In [8], a very general existence result for rate-independent processes is proved (with coercive surface energy density). Then, one should carry the information coming from the displacement history over to the continuous formulation.

The paper is organized as follows. In Section 1, we introduce the problem and in Section 2 give the main properties of the time discrete evolution. We show that our formulation is well-posed, i.e. it admits a solution and the solution is unique. In Section 3, we derive the a priori estimate used in performing the limit as the time step goes to zero. In Section 4, we present the time continuous formulation of the problem that is our main result.

\section{The VARIATIONAL FORMUlation}

In [11], the debonding of an inextensible and perfectly flexible thin layer due to a cyclic deflection is considered. Let us briefly recall the problem.

At the initial time $t=0$ the layer is perfectly glued to a rigid substrate. One end is submitted to a constant horizontal tension $N$ and to a vertical cyclic deflection $V(t)$, while the other end is fixed.

Let $u(t, x)$ be the displacement of the layer at time $t$ and let $L$ be the length of the layer. Following the idea introduced by Francfort and Marigo [7], the quasi-static evolution of the debonding is the result of a limit process of a time discretized sequence of minimization problems. At each time $t$, the total energy to be minimized is the result of the competition between potential and surface energy.

Since the layer is inextensible and perfectly flexible, the potential energy can be written as

$$
\frac{N}{2} \int_{0}^{L}\left|\frac{\partial u}{\partial x}(t, x)\right|^{2} \mathrm{~d} x .
$$

Meanwhile, the surface energy density is a generic increase concave function $\phi$ (in [11], the case of Dugdale energy density is presented). In order to account for the fatigue, a memory field $\delta(x, t):=\int_{0}^{t}[\dot{u}(\tau, x)]^{+} \mathrm{d} \tau$ is introduced in [11].

The surface energy can be written as

$$
\int_{0}^{L} \phi(\delta(t, x)) \mathrm{d} x
$$

Specifically, the problem can be stated as follows.

Let $N$ be a positive constant, let $\phi:[0, \infty) \rightarrow[0, \infty)$ be a non-decreasing concave function ${ }^{1}$, with $\phi(0)=0$, $\phi$ is differentiable at 0 and $\phi^{\prime}(0)<+\infty$, and let $V:[0, T] \rightarrow[0, \infty), V(0)=0$, be an absolutely continuous function with piecewise continuous derivative. We assume that $V$ has a finite number of cycles, meaning that the open set $\{t \in(0, T): \dot{V}$ is continuous at $\mathrm{t}, \dot{V}(t)>0\}$ has finitely many connected components.

By the concavity assumption, the left and the right derivative of $\phi$ exist finite everywhere in $(0, \infty)$. We denote by $\phi_{-}^{\prime}$ the left derivative of $\phi$ at any point in $(0, \infty)$. At the point 0 , the derivative of $\phi$ is nothing but its

\footnotetext{
${ }^{1} \phi$ is in particular sub-additive.
} 
right derivative, since $\phi$ is not defined in a left neighbourhood of 0 . With an abuse of notation, we still denote the derivative of $\phi$ in 0 by $\phi_{-}^{\prime}(0):=\phi^{\prime}(0)$.

The starting point of the formulation is the discretization in time.

Let $0=t_{0}^{n}<t_{1}^{n}<\cdots<t_{p(n)}^{n}=T$ be any given partition $\mathcal{P}^{n}$ of the interval $[0, T]$. For any $i$ in $\{0,1, \ldots, p(n)\}$, the problem of the debonding of a thin layer is defined by iteration as follows:

$\left(\mathbf{P}_{i}^{n}\right)$ minimize

$$
\mathcal{I}_{i}^{n}(u):=\frac{N}{2} \int_{0}^{L}\left|\frac{\mathrm{d} u}{\mathrm{~d} x}\right|^{2}+\int_{0}^{L} \phi\left(\delta_{i-1}^{n}+\left[u-u_{i-1}^{n}\right]^{+}\right)
$$

on the set of functions $u$ in $\mathbf{W}^{1,2}(0, L)$ satisfying $u(0)=0, u(L)=V\left(t_{i}^{n}\right)=: V_{i}^{n}$, where

$$
\begin{aligned}
u_{i-1}^{n} & := \begin{cases}\text { a solution to }\left(\mathbf{P}_{i-1}^{n}\right), & i \geq 1 \\
0, & i=0,\end{cases} \\
\delta_{i-1}^{n}: & = \begin{cases}\sum_{k=0}^{i-1}\left[u_{k}^{n}-u_{k-1}^{n}\right]^{+}, & i \geq 1 \\
0, & i=0 .\end{cases}
\end{aligned}
$$

\section{The time-Discrete Evolution}

First of all, observe that any $\left(\mathbf{P}_{i}^{n}\right)$ does admit a solution. In fact, its related Lagrangian

$$
L_{i}^{n}(x, u, \xi):=\frac{N}{2}|\xi|^{2}+\phi\left(\delta_{i-1}^{n}(x)+\left[u-u_{i-1}^{n}(x)\right]^{+}\right)
$$

is coercive and continuous. From standard arguments of the Direct Method of the Calculus of Variations [2], it follows that it admits at least a solution $u_{i}^{n}$ in $\mathbf{W}^{1,2}(0, L)$, for any given boundary conditions.

Any solution $u_{i}^{n}$ satisfies the Euler-Lagrange equation in a weak form. This is proved in the following lemma.

Lemma 1. For any $i$ in $\{0,1, \ldots, p(n)\}, u_{i}^{n}$ satisfies the Euler-Lagrange condition:

$$
0 \leq N \int_{0}^{L} \frac{\mathrm{d} u_{i}^{n}}{\mathrm{~d} x} \frac{\mathrm{d} v_{0}}{\mathrm{~d} x}+\int_{\left\{u_{i}^{n}>u_{i-1}^{n}\right\}} \phi_{-}^{\prime}\left(\delta_{i}^{n}\right) v_{0}+\int_{\left\{u_{i}^{n}=u_{i-1}^{n}\right\}} \phi_{+}^{\prime}\left(\delta_{i-1}^{n}\right)\left[v_{0}\right]^{+},
$$

for any $v_{0}$ in $\mathbf{W}_{0}^{1,2}(0, L)$.

Proof. Fix $v_{0}$ in $\mathbf{W}_{0}^{1,2}(0, L), \epsilon>0$ and consider the function $u_{i}^{n}+\epsilon v_{0}$. By the minimality of $u_{i}^{n}$, we have

$$
\begin{aligned}
0 \leq \mathcal{I}_{i}^{n}\left(u_{i}^{n}+\epsilon v_{0}\right)-\mathcal{I}_{i}^{n}\left(u_{i}^{n}\right)= & \epsilon^{2} \frac{N}{2} \int_{0}^{L}\left|\frac{\mathrm{d} v_{0}}{\mathrm{~d} x}\right|^{2}+\epsilon N \int_{0}^{L} \frac{\mathrm{d} u_{i}^{n}}{\mathrm{~d} x} \frac{\mathrm{d} v_{0}}{\mathrm{~d} x} \\
& +\int_{0}^{L} \phi\left(\delta_{i-1}^{n}+\left[u_{i}^{n}+\epsilon v_{0}-u_{i-1}^{n}\right]^{+}\right)-\int_{0}^{L} \phi\left(\delta_{i-1}^{n}+\left[u_{i}^{n}-u_{i-1}^{n}\right]^{+}\right) .
\end{aligned}
$$

The Euler-Lagrange condition is obtained taking the limit in the previous inequality as $\epsilon$ goes to 0 :

$$
0 \leq N \int_{0}^{L} \frac{\mathrm{d} u_{i}^{n}}{\mathrm{~d} x} \frac{\mathrm{d} v_{0}}{\mathrm{~d} x}+\liminf _{\epsilon \rightarrow 0^{+}} \frac{1}{\epsilon}\left\{\int_{0}^{L} \phi\left(\delta_{i-1}^{n}+\left[u_{i}^{n}+\epsilon v_{0}-u_{i-1}^{n}\right]^{+}\right)-\int_{0}^{L} \phi\left(\delta_{i-1}^{n}+\left[u_{i}^{n}-u_{i-1}^{n}\right]^{+}\right)\right\}
$$


The concavity of $\phi$ implies that $\phi_{-}^{\prime}$ is bounded by $\phi^{\prime}(0)$; we can therefore apply the Lebesgue Dominated Convergence Theorem and it follows that

$$
\begin{aligned}
& \liminf _{\epsilon \rightarrow 0^{+}} \frac{1}{\epsilon}\left\{\int_{0}^{L} \phi\left(\delta_{i-1}^{n}+\left[u_{i}^{n}+\epsilon v_{0}-u_{i-1}^{n}\right]^{+}\right)-\int_{0}^{L} \phi\left(\delta_{i-1}^{n}+\left[u_{i}^{n}-u_{i-1}^{n}\right]^{+}\right)\right\} \\
& \quad=\int_{0}^{L} \lim _{\epsilon \rightarrow 0^{+}} \frac{1}{\epsilon}\left\{\phi\left(\delta_{i-1}^{n}+\left[u_{i}^{n}+\epsilon v_{0}-u_{i-1}^{n}\right]^{+}\right)-\phi\left(\delta_{i-1}^{n}+\left[u_{i}^{n}-u_{i-1}^{n}\right]^{+}\right)\right\} \\
&=\int_{\left\{u_{i}^{n}-u_{i-1}^{n}>0, v_{0} \geq 0\right\}} \phi_{+}^{\prime}\left(\delta_{i}^{n}\right) v_{0}+\int_{\left\{u_{i}^{n}-u_{i-1}^{n}>0, v_{0}<0\right\}} \phi_{-}^{\prime}\left(\delta_{i}^{n}\right) v_{0}+\int_{\left\{u_{i}^{n}-u_{i-1}^{n}=0\right\}} \phi_{+}^{\prime}\left(\delta_{i-1}^{n}\right)\left[v_{0}\right]^{+} \\
& \leq \int_{\left\{u_{i}^{n}>u_{i-1}^{n}\right\}} \phi_{-}^{\prime}\left(\delta_{i}^{n}\right) v_{0}+\int_{\left\{u_{i}^{n}=u_{i-1}^{n}\right\}} \phi_{+}^{\prime}\left(\delta_{i-1}^{n}\right)\left[v_{0}\right]^{+}
\end{aligned}
$$

where we used the fact that $\phi_{+}^{\prime} \leq \phi_{-}^{\prime}$ to estimate the integral on the set $\left\{u_{i}^{n}-u_{i-1}^{n}>0, v_{0} \geq 0\right\}$. That concludes the proof.

Two weaker forms of the Euler-Lagrange condition will be used in the paper. We recall that the concavity of $\phi$ implies that $\phi_{-}^{\prime}$ is non-negative and $\phi_{+}^{\prime} \leq \phi_{-}^{\prime}$. First

$$
0 \leq N \int_{0}^{L} \frac{\mathrm{d} u_{i}^{n}}{\mathrm{~d} x} \frac{\mathrm{d} v_{0}}{\mathrm{~d} x}+\int_{0}^{L} \phi_{-}^{\prime}\left(\delta_{i}^{n}\right) v_{0},
$$

for any $v_{0} \geq 0, v_{0} \in \mathbf{W}_{0}^{1,2}(0, L)$.

Then, considering variations $v_{0}$ that vanish on the set $\left\{u_{i}^{n}=u_{i-1}^{n}\right\}$, we obtain the equation

$$
0=N \int_{0}^{L} \frac{\mathrm{d} u_{i}^{n}}{\mathrm{~d} x} \frac{\mathrm{d} v_{0}}{\mathrm{~d} x}+\int_{\left\{u_{i}^{n}>u_{i-1}^{n}\right\}} \phi_{-}^{\prime}\left(\delta_{i}^{n}\right) v_{0},
$$

for any $v_{0} \in \mathbf{W}_{0}^{1,2}(0, L),\left.v_{0}\right|_{\left\{u_{i}^{n}=u_{i-1}^{n}\right\}}=0$, by writing the Euler-Lagrange condition for $v_{0}$ and for $-v_{0}$. In what follows, we refer to the this equation has the Euler-Lagrange equation.

Lemma 2. The $u_{i}^{n}$ enjoy the following properties:

I. $u_{i}^{n}$ are convex;

II. $u_{i}^{n}$ belong to $\mathbf{W}^{2, \infty}(0, L)$;

III. if $x$ in $[0, L]$ is such that $u_{i}^{n}(x)=0$, then $u_{i}^{n}=0$ on $[0, x]$ ( $\Rightarrow u_{i}^{n}$ are non-negative);

IV. $u_{i}^{n}$ are strictly increasing in $\left\{x \in[0, L]: u_{i}^{n}>0\right\}=\left(x_{i}^{n}, L\right]$.

Proof of I. Suppose that $u_{i}^{n}$ is not convex.

Recall that $u_{i}^{n}$ is continuous in $[0, L]$ since it belongs to $\mathbf{W}^{1,2}(0, L)$. Hence, there exists an interval $[a, b] \subset$ $[0, L]$ such that $u_{i}^{n}$ is above the line segment that joint $u_{i}^{n}(a)$ to $u_{i}^{n}(b)$, i.e.

$$
u_{i}^{n}(x)>u_{i}^{n}(a)+(x-a) \frac{u_{i}^{n}(b)-u_{i}^{n}(a)}{b-a}=: r_{a, b}(x),
$$

for any $x$ in $(a, b)$.

Consider the admissible competitor $\bar{u}$ to $u_{i}^{n}$ given by

$$
\bar{u}:=r_{a, b} \chi_{[a, b]}+u_{i}^{n}\left\{1-\chi_{[a, b]}\right\} .
$$


We have that

$$
\mathcal{I}_{i}^{n}\left(u_{i}^{n}\right)-\mathcal{I}_{i}^{n}(\bar{u})=\frac{N}{2} \int_{a}^{b}\left|\frac{\mathrm{d} u_{i}^{n}}{\mathrm{~d} x}\right|^{2}+\int_{a}^{b} \phi\left(\delta_{i}^{n}\right)-\frac{N}{2} \frac{\left|u_{i}^{n}(b)-u_{i}^{n}(a)\right|^{2}}{b-a}-\int_{a}^{b} \phi\left(\delta_{i-1}^{n}+\left[r_{a, b}-u_{i-1}^{n}\right]^{+}\right) .
$$

By the monotonicity of $\phi$ and Jensen's inequality, $\mathcal{I}_{i}^{n}\left(u_{i}^{n}\right)-\mathcal{I}_{i}^{n}(\bar{u})>0$, and, hence, $u_{i}^{n}$ would not be a minimizer.

Proof of II. Let $x_{1}, x_{2}$ be two points in $(0, L), x_{1} \leq x_{2}$ (notice that they might be the same point). Fix $\epsilon>0$, $\epsilon \leq \min \left\{x_{1}, L-x_{2}\right\}$, and set

$$
\frac{\mathrm{d} v_{0}^{n}}{\mathrm{~d} x}(x):=\left\{\begin{array}{ll}
0, & x \in\left[0, x_{1}-\epsilon\right) \\
1 / \epsilon, & x \in\left[x_{1}-\epsilon, x_{1}\right) \\
0, & x \in\left[x_{1}, x_{2}\right) \\
-1 / \epsilon, & x \in\left[x_{2}, x_{2}+\epsilon\right) \\
0, & x \in\left[x_{2}+\epsilon, L\right]
\end{array} \quad, \quad v_{0}^{n}(x):=\int_{0}^{x} \frac{\mathrm{d} v_{0}^{n}}{\mathrm{~d} x}=\left\{\begin{array}{ll}
0, & x \in\left[0, x_{1}-\epsilon\right) \\
\left(x-x_{1}+\epsilon\right] / \epsilon, & x \in\left[x_{1}-\epsilon, x_{1}\right) \\
1, & x \in\left[x_{1}, x_{2}\right) \\
\left(x_{2}+\epsilon-x\right) / \epsilon, & x \in\left[x_{2}, x_{2}+\epsilon\right) \\
0, & x \in\left[x_{2}+\epsilon, L\right]
\end{array} .\right.\right.
$$

By definition, $v_{0}^{n}$ belongs to $\mathbf{W}_{0}^{1,2}(0, L)$.

By Lemma 1, $u_{i}^{n}$ satisfies the Euler-Lagrange inequality (1) in direction $v_{0}^{n}$ :

$$
\begin{aligned}
0 & \leq N \int_{x_{1}-\epsilon}^{x_{1}} \frac{1}{\epsilon} \frac{\mathrm{d} u_{i}^{n}}{\mathrm{~d} x}-N \int_{x_{2}}^{x_{2}+\epsilon} \frac{1}{\epsilon} \frac{\mathrm{d} u_{i}^{n}}{\mathrm{~d} x}+\int_{x_{1}-\epsilon}^{x_{1}} \phi_{-}^{\prime}\left(\delta_{i}^{n}\right) \frac{x-x_{1}+\epsilon}{\epsilon}+\int_{x_{1}}^{x_{2}} \phi_{-}^{\prime}\left(\delta_{i}^{n}\right)+\int_{x_{2}}^{x_{2}+\epsilon} \phi_{-}^{\prime}\left(\delta_{i}^{n}\right) \frac{x_{2}+\epsilon-x}{\epsilon} \\
& \leq N \frac{u_{i}^{n}\left(x_{1}\right)-u_{i}^{n}\left(x_{1}-\epsilon\right)}{\epsilon}-N \frac{u_{i}^{n}\left(x_{2}+\epsilon\right)-u_{i}^{n}\left(x_{2}\right)}{\epsilon}+\phi^{\prime}(0)\left(x_{2}-x_{1}+2 \epsilon\right) .
\end{aligned}
$$

As $\epsilon$ goes to 0 , using the convexity of $u_{i}^{n}$ (point I), we obtain

$$
N\left\{\frac{\mathrm{d} u_{i}^{n}}{\mathrm{~d} x^{+}}\left(x_{2}\right)-\frac{\mathrm{d} u_{i}^{n}}{\mathrm{~d} x^{-}}\left(x_{1}\right)\right\}=N\left|\frac{\mathrm{d} u_{i}^{n}}{\mathrm{~d} x^{+}}\left(x_{2}\right)-\frac{\mathrm{d} u_{i}^{n}}{\mathrm{~d} x^{-}}\left(x_{1}\right)\right| \leq \phi^{\prime}(0)\left(x_{2}-x_{1}\right) .
$$

By letting $x_{1}=x_{2}$ vary in $(0, L)$, it follows that the right and the left derivative of $u_{i}^{n}$ coincide, i.e. $\frac{\mathrm{d} u_{i}^{n}}{\mathrm{~d} x^{+}}=\frac{\mathrm{d} u_{i}^{n}}{\mathrm{~d} x^{-}}$. Hence,

$$
\left|\frac{\mathrm{d} u_{i}^{n}}{\mathrm{~d} x}\left(x_{2}\right)-\frac{\mathrm{d} u_{i}^{n}}{\mathrm{~d} x}\left(x_{1}\right)\right| \leq \frac{\phi^{\prime}(0)}{N}\left(x_{2}-x_{1}\right),
$$

that implies, since $x_{1}$ and $x_{2}$ are arbitrary in $(0, L), \frac{\mathrm{d} u_{i}^{n}}{\mathrm{~d} x} \in \mathbf{W}^{1, \infty}(0, L)$.

Proof of III. Consider the admissible competitor $\bar{u}$ to $u_{i}^{n}$ given by $\bar{u}:=u_{i}^{n} \chi_{[x, L]}$. We proceed by induction on $i$. In case $i=0$, by the minimality of $u_{0}^{n}$, we have that

$$
0 \geq \mathcal{I}_{0}^{n}\left(u_{0}^{n}\right)-\mathcal{I}_{0}^{n}(\bar{u})=\frac{N}{2} \int_{0}^{x}\left|\frac{\mathrm{d} u_{0}^{n}}{\mathrm{~d} x}\right|^{2}+\int_{0}^{x} \phi\left(\delta_{0}^{n}\right) \geq \frac{N}{2} \int_{0}^{x}\left|\frac{\mathrm{d} u_{0}^{n}}{\mathrm{~d} x}\right|^{2} .
$$

Hence, $\frac{\mathrm{d} u_{0}^{n}}{\mathrm{~d} x}=0$ and, by the fact $u_{0}^{n}(0)=0, u_{0}^{n}=0$ on $[0, x]$.

In case $i \geq 1$, assume that the statement is true for $u_{i-1}^{n}$. Then, $u_{i-1}^{n} \geq 0$ and

$$
\begin{aligned}
0 \geq \mathcal{I}_{i}^{n}\left(u_{i}^{n}\right)-\mathcal{I}_{i}^{n}(\bar{u}) & =\frac{N}{2} \int_{0}^{x}\left|\frac{\mathrm{d} u_{i}^{n}}{\mathrm{~d} x}\right|^{2}+\int_{0}^{x} \phi\left(\delta_{i}^{n}\right)-\int_{0}^{x} \phi\left(\delta_{i-1}^{n}+\left[-u_{i-1}^{n}\right]^{+}\right) \\
& =\frac{N}{2} \int_{0}^{x}\left|\frac{\mathrm{d} u_{i}^{n}}{\mathrm{~d} x}\right|^{2}+\int_{0}^{x} \phi\left(\delta_{i}^{n}\right)-\int_{0}^{x} \phi\left(\delta_{i-1}^{n}\right) \geq \frac{N}{2} \int_{0}^{x}\left|\frac{\mathrm{d} u_{i}^{n}}{\mathrm{~d} x}\right|^{2},
\end{aligned}
$$


by the monotonicity of $\phi$ (by definition, $\delta_{i}^{n} \geq \delta_{i-1}^{n}$, for any $i$ ). Hence, $\frac{\mathrm{d} u_{i}^{n}}{\mathrm{~d} x}=0$ and, by the fact $u_{i}^{n}(0)=0$, $u_{i}^{n}=0$ on $[0, x]$.

Proof of IV. By point III, there exists $x_{i}^{n}$ in $[0, L)$ such that $\left\{x \in[0, L]: u_{i}^{n}>0\right\}=\left(x_{i}^{n}, L\right]$. Since $u_{i}^{n}\left(x_{i}^{n}\right)=0$ and $u_{i}^{n}>0$ in $\left(x_{i}^{n}, L\right]$, by the convexity of $u_{i}^{n}$ (point I), it follows that $\frac{\mathrm{d} u_{i}^{n}}{\mathrm{~d} x}>0$ in $\left(x_{i}^{n}, L\right]$.

Any problem $\left(\mathbf{P}_{i}^{n}\right)$ is well-posed. In fact, it admits a solution (see the first observation at the beginning of this section) and the solution is unique. That is proved in Theorem 1 below.

Theorem 1. For any $i$ in $\{0,1, \ldots, p(n)\},\left(\mathbf{P}_{i}^{n}\right)$ admits unique solution.

Proof. We prove the result by iteration on $i$.

Consider the problem $\left(\mathbf{P}_{0}^{n}\right)$.

In case $V_{0}^{n}=0$, by point III of Lemma $2, u_{0}^{n}=0$ is the unique solution.

In case $V_{0}^{n}>0$, suppose that there exist two solutions $\bar{w}_{0}^{n} \neq \bar{u}_{0}^{n}$. From the continuity of $\bar{w}_{0}^{n}$ and $\bar{u}_{0}^{n}$ (point II, Lemma 2), we can construct other two solutions $w_{0}^{n} \neq u_{0}^{n}$ such that $w_{0}^{n} \geq u_{0}^{n}$. They are defined by $w_{0}^{n}:=\max \left\{\bar{w}_{0}^{n}, \bar{u}_{0}^{n}\right\}$ and $u_{0}^{n}:=\min \left\{\bar{w}_{0}^{n}, \bar{u}_{0}^{n}\right\}$. The fact that they are solutions comes from the global character of minima. More precisely, if $a$ and $b$ are two points in $[0, L], a<b$, such that $\bar{u}_{0}^{n}(a)=\bar{w}_{0}^{n}(a)$ and $\bar{u}_{0}^{n}(b)=\bar{w}_{0}^{n}(b)$, then, by comparing $\mathcal{I}_{0}^{n}\left(\bar{u}_{0}^{n}\right)$ to $\mathcal{I}_{0}^{n}\left(\bar{u}_{0}^{n}\left(1-\chi_{[a, b]}\right)+\bar{w}_{0}^{n} \chi_{[a, b]}\right)$ and $\mathcal{I}_{0}^{n}\left(\bar{w}_{0}^{n}\right)$ to $\mathcal{I}_{0}^{n}\left(\bar{w}_{0}^{n}\left(1-\chi_{[a, b]}\right)+\bar{u}_{0}^{n} \chi_{[a, b]}\right)$, we get $\left.\mathcal{I}_{0}^{n}\right|_{[a, b]}\left(\bar{u}_{0}^{n}\right)=\left.\mathcal{I}_{0}^{n}\right|_{[a, b]}\left(\bar{w}_{0}^{n}\right)$, where $\left.\mathcal{I}_{0}^{n}\right|_{[a, b]}$ denotes the functional $\mathcal{I}_{0}^{n}$ in which the integrals are taken on $[a, b]$.

Let $y_{0}^{n}$ and $x_{0}^{n}$ be the points defined as

$$
\begin{aligned}
& y_{0}^{n}:=\sup \left\{x \in[0, L]: w_{0}^{n}(x)=0\right\} \\
& x_{0}^{n}:=\sup \left\{x \in[0, L]: u_{0}^{n}(x)=0\right\} .
\end{aligned}
$$

Point III of Lemma 2 implies that $\left\{x \in[0, L]: w_{0}^{n}(x)=0\right\}=\left[0, y_{0}^{n}\right],\left\{x \in[0, L]: u_{0}^{n}(x)=0\right\}=\left[0, x_{0}^{n}\right]$, and, from the assumption $w_{0}^{n} \geq u_{0}^{n}$, it follows that $y_{0}^{n} \leq x_{0}^{n}$. Furthermore, by the continuity of the derivative of $u_{0}^{n}$ and of $w_{0}^{n}$ (Lem. 2, point II), it follows that

$$
0=w_{i}^{n}\left(y_{0}^{n}\right)=u_{i}^{n}\left(x_{0}^{n}\right)=\frac{\mathrm{d} w_{i}^{n}}{\mathrm{~d} x}\left(y_{0}^{n}\right)=\frac{\mathrm{d} u_{i}^{n}}{\mathrm{~d} x}\left(x_{0}^{n}\right) .
$$

From the definition of $x_{0}^{n}$ and $y_{0}^{n}$, we obtain from Euler-Lagrange equation (2)

$$
0=N \int_{x_{0}^{n}}^{L} \frac{\mathrm{d} u_{0}^{n}}{\mathrm{~d} x} \frac{\mathrm{d} v_{0}}{\mathrm{~d} x}+\int_{x_{0}^{n}}^{L} \phi_{-}^{\prime}\left(u_{0}^{n}\right) v_{0}, \quad \text { for any } v_{0} \in \mathbf{W}_{0}^{1,1}\left(x_{0}^{n}, L\right),
$$

and,

$$
0=N \int_{y_{0}^{n}}^{L} \frac{\mathrm{d} w_{i}^{n}}{\mathrm{~d} x} \frac{\mathrm{d} v_{0}}{\mathrm{~d} x}+\int_{y_{0}^{n}}^{L} \phi_{-}^{\prime}\left(w_{0}^{n}\right) v_{0}, \quad \text { for any } v_{0} \in \mathbf{W}_{0}^{1,1}\left(y_{0}^{n}, L\right) .
$$

By the fact that $\frac{\mathrm{d} u_{i}^{n}}{\mathrm{~d} x}$ and $\frac{\mathrm{d} w_{i}^{n}}{\mathrm{~d} x}$ belong to $\mathbf{W}^{1,1}(0, L)$ (Lem. 2, point II), using integration by part formula and the Fundamental Lemma of the Calculus of Variations [2], we can write both equations in a classical form:

$$
\begin{aligned}
& N \frac{\mathrm{d}^{2} u_{0}^{n}}{\mathrm{~d} x^{2}}(x)=\phi_{-}^{\prime}\left(u_{0}^{n}(x)\right), \quad \text { for a.e. } x \in\left[x_{0}^{n}, L\right], \\
& N \frac{\mathrm{d}^{2} w_{0}^{n}}{\mathrm{~d} x^{2}}(x)=\phi_{-}^{\prime}\left(w_{0}^{n}(x)\right), \quad \text { for a.e. } x \in\left[y_{0}^{n}, L\right] .
\end{aligned}
$$


Since, from point IV of Lemma $2, w_{0}^{n}$ and $u_{0}^{n}$ are strictly increasing respectively in $\left[y_{0}^{n}, L\right]$ and $\left[x_{0}^{n}, L\right]$, their inverse functions $w_{0}^{n,-1}$ and $u_{0}^{n,-1}$ are well defined in $\left[0, V_{0}^{n}\right]$. As we plug the inverse functions in the equations, we obtain

$$
N \frac{\mathrm{d}^{2} u_{0}^{n}}{\mathrm{~d} x^{2}}\left(u_{0}^{n,-1}(z)\right)=\phi^{\prime}(z)=N \frac{\mathrm{d}^{2} w_{0}^{n}}{\mathrm{~d} x^{2}}\left(w_{0}^{n,-1}(z)\right),
$$

for any $z$ in $\left[0, V_{0}^{n}\right]$. That gives rise to a contradiction.

Indeed, fix $x$ in $\left(x_{0}^{n}, L\right]$. By integrating over $\left[0, u_{0}^{n}(x)\right]$ the chain of equalities above and applying the changes of variable $z=u_{0}^{n}(y)$ and $z=w_{0}^{n}(y)$, we obtain

$$
\begin{aligned}
N \int_{x_{0}^{n}}^{x} \frac{\mathrm{d}^{2} u_{0}^{n}}{\mathrm{~d} x^{2}}(y) \frac{\mathrm{d} u_{0}^{n}}{\mathrm{~d} x}(y) \mathrm{d} y & =N \int_{0}^{u_{0}^{n}(x)} \frac{\mathrm{d}^{2} u_{0}^{n}}{\mathrm{~d} x^{2}}\left(u_{0}^{n,-1}(z)\right) \mathrm{d} z \\
& =N \int_{0}^{u_{0}^{n}(x)} \frac{\mathrm{d}^{2} w_{0}^{n}}{\mathrm{~d} x^{2}}\left(w_{0}^{n,-1}(z)\right) \mathrm{d} z=N \int_{y_{0}^{n}}^{w_{0}^{n,-1}\left(u_{0}^{n}(x)\right)} \frac{\mathrm{d}^{2} w_{0}^{n}}{\mathrm{~d} x^{2}}(y) \frac{\mathrm{d} w_{0}^{n}}{\mathrm{~d} x}(y) \mathrm{d} y .
\end{aligned}
$$

Since $\frac{1}{2}\left|\frac{\mathrm{d} u_{0}^{n}}{\mathrm{~d} x}\right|^{2}$ is a primitive of $\frac{\mathrm{d}^{2} u_{0}^{n}}{\mathrm{~d} x^{2}} \frac{\mathrm{d} u_{0}^{n}}{\mathrm{~d} x}$ (and analogously for $w_{0}^{n}$ ), it follows

$$
\begin{aligned}
\frac{N}{2}\left|\frac{\mathrm{d} u_{0}^{n}}{\mathrm{~d} x}(x)\right|^{2} & =\frac{N}{2}\left|\frac{\mathrm{d} u_{0}^{n}}{\mathrm{~d} x}(x)\right|^{2}-\frac{N}{2}\left|\frac{\mathrm{d} u_{0}^{n}}{\mathrm{~d} x}\left(x_{0}^{n}\right)\right|^{2} \\
& =\frac{N}{2}\left|\frac{\mathrm{d} w_{0}^{n}}{\mathrm{~d} x}\left(w_{0}^{n,-1}\left(u_{0}^{n}(x)\right)\right)\right|^{2}-\frac{N}{2}\left|\frac{\mathrm{d} w_{0}^{n}}{\mathrm{~d} x}\left(y_{0}^{n}\right)\right|^{2}=\frac{N}{2}\left|\frac{\mathrm{d} w_{0}^{n}}{\mathrm{~d} x}\left(w_{0}^{n,-1}\left(u_{0}^{n}(x)\right)\right)\right|^{2}
\end{aligned}
$$

(we recall that $\left.\frac{\mathrm{d} u_{0}^{n}}{\mathrm{~d} x}\left(x_{0}^{n}\right)=\frac{\mathrm{d} w_{0}^{n}}{\mathrm{~d} x}\left(y_{0}^{n}\right)=0\right)$.

Observe that $u_{0}^{n}(x) \leq w_{0}^{n}(x), w_{0}^{n,-1}\left(u_{0}^{n}(x)\right) \leq x$. Then, by the convexity of $w_{0}^{n}$,

$$
\frac{\mathrm{d} u_{0}^{n}}{\mathrm{~d} x}(x)=\frac{\mathrm{d} w_{0}^{n}}{\mathrm{~d} x}\left(w_{0}^{n,-1}\left(u_{0}^{n}(x)\right)\right) \leq \frac{\mathrm{d} w_{0}^{n}}{\mathrm{~d} x}(x) .
$$

Since $u_{0}^{n} \neq w_{0}^{n}$, there exists at least a point $x^{n}$ in $\left(x_{0}^{n}, L\right]$ with $u_{0}^{n}\left(x^{n}\right)<w_{0}^{n}\left(x^{n}\right)$. But from the above inequality,

$$
V_{0}^{n}-u_{0}^{n}\left(x^{n}\right)=\int_{x^{n}}^{L} \frac{\mathrm{d} u_{0}^{n}}{\mathrm{~d} x} \leq \int_{x^{n}}^{L} \frac{\mathrm{d} w_{0}^{n}}{\mathrm{~d} x}=V_{0}^{n}-w_{0}^{n}\left(x^{n}\right),
$$

and, hence, $w_{0}^{n}\left(x^{n}\right) \leq u_{0}^{n}\left(x^{n}\right)$, a contradiction. Therefore, we conclude that there cannot exist two distinct solutions to $\left(\mathbf{P}_{0}^{n}\right)$.

Assume that $\left(\mathbf{P}_{i-1}^{n}\right)$ admits unique solution $u_{i-1}^{n}$ and consider the problem $\left(\mathbf{P}_{i}^{n}\right)$.

We claim that, if $u_{i}^{n}(x)=u_{i-1}^{n}(x)$ at a point $x \in[0, L)$, then $u_{i}^{n}=u_{i-1}^{n}$ in the entire interval $[0, x]$. This is a consequence of the uniqueness of $u_{i-1}^{n}$. Indeed, consider the competitors given by $\bar{u}_{i}^{n}:=u_{i-1}^{n} \chi_{[0, x]}+u_{i}^{n} \chi_{(x, L]}$ and $\bar{u}_{i-1}^{n}:=u_{i}^{n} \chi_{[0, x]}+u_{i-1}^{n} \chi_{(x, L]}$. Then,

$$
\begin{aligned}
& 0 \leq \mathcal{I}_{i}^{n}\left(\bar{u}_{i}^{n}\right)-\mathcal{I}_{i}^{n}\left(u_{i}^{n}\right)=\left.\mathcal{I}_{i}^{n}\right|_{[0, x]}\left(u_{i-1}^{n}\right)-\left.\mathcal{I}_{i}^{n}\right|_{[0, x]}\left(u_{i}^{n}\right), \\
& 0 \leq \mathcal{I}_{i-1}^{n}\left(\bar{u}_{i-1}^{n}\right)-\mathcal{I}_{i-1}^{n}\left(u_{i-1}^{n}\right)=\left.\mathcal{I}_{i-1}^{n}\right|_{[0, x]}\left(u_{i}^{n}\right)-\left.\mathcal{I}_{i-1}^{n}\right|_{[0, x]}\left(u_{i-1}^{n}\right) .
\end{aligned}
$$

It follows directly from the sub-additivity of $\phi$ and of $[\cdot]^{+}$that

$$
\begin{aligned}
\left.\mathcal{I}_{i-1}^{n}\right|_{[0, x]}\left(u_{i}^{n}\right)=\frac{N}{2} \int_{0}^{x}\left|\frac{\mathrm{d} u_{i}^{n}}{\mathrm{~d} x}\right|^{2}+ & \int_{0}^{x} \phi\left(\delta_{i-2}^{n}+\left[u_{i}^{n}-u_{i-2}^{n}\right]^{+}\right) \\
& \leq \frac{N}{2} \int_{0}^{x}\left|\frac{\mathrm{d} u_{i}^{n}}{\mathrm{~d} x}\right|^{2}+\int_{0}^{x} \phi\left(\delta_{i-2}^{n}+\left[u_{i}^{n}-u_{i-1}^{n}\right]+\left[u_{i-1}^{n}-u_{i-2}^{n}\right]^{+}\right) \leq\left.\mathcal{I}_{i}^{n}\right|_{[0, x]}\left(u_{i}^{n}\right),
\end{aligned}
$$


and, from the definition of $\delta_{i}^{n}$, that

$$
\begin{aligned}
\left.\mathcal{I}_{i-1}^{n}\right|_{[0, x]}\left(u_{i-1}^{n}\right)=\frac{N}{2} \int_{0}^{x}\left|\frac{\mathrm{d} u_{i-1}^{n}}{\mathrm{~d} x}\right|^{2}+\int_{0}^{x} \phi\left(\delta_{i-1}^{n}\right) \\
\quad=\frac{N}{2} \int_{0}^{x}\left|\frac{\mathrm{d} u_{i-1}^{n}}{\mathrm{~d} x}\right|^{2}+\int_{0}^{x} \phi\left(\delta_{i-1}^{n}+\left[u_{i-1}^{n}-u_{i-1}^{n}\right]^{+}\right)=\left.\mathcal{I}_{i}^{n}\right|_{[0, x]}\left(u_{i-1}^{n}\right) .
\end{aligned}
$$

Therefore, $\mathcal{I}_{i-1}^{n}\left(\bar{u}_{i-1}^{n}\right)=\mathcal{I}_{i-1}^{n}\left(u_{i-1}^{n}\right)$, that implies, by the uniqueness of $u_{i-1}^{n}, \bar{u}_{i-1}^{n}=u_{i-1}^{n}$ and, by the definition of $\bar{u}_{i-1}^{n}, u_{i}^{n}=u_{i-1}^{n}$ in $[0, x]$. As a consequence, we obtain that $u_{i}^{n} \geq u_{i-1}^{n}$ on $[0, L]$.

In case $V_{i}^{n} \leq V_{i-1}^{n}$, problem $\left(\mathbf{P}_{i}^{n}\right)$ is equivalent to minimize

$$
\frac{N}{2} \int_{0}^{L}\left|\frac{\mathrm{d} u}{\mathrm{~d} x}\right|^{2}+\int_{0}^{L} \phi\left(\delta_{i-1}^{n}\right)=\frac{N}{2} \int_{0}^{L}\left|\frac{\mathrm{d} u}{\mathrm{~d} x}\right|^{2}+\text { const. }
$$

on the set of functions $u$ of $\mathbf{W}^{1,2}(0, L)$ satisfying $u(0)=0, u(L)=V_{i}^{n}$ and $u \leq u_{i-1}^{n}$. That is an obstacle problem with convex obstruction $u_{i-1}^{n}$ (Lem. 2, point III). The unique solution is

$$
u_{i}^{n}(x)= \begin{cases}u_{i-1}^{n}(x), & x \in\left[0, x_{i}^{n}\right], \\ \left(x-x_{i}^{n}\right)\left\{V_{i}^{n}-u_{i-1}^{n}\left(x_{i}^{n}\right)\right\} /\left(L-x_{i}^{n}\right)+u_{i-1}^{n}\left(x_{i}^{n}\right), & x \in\left(x_{i}^{n}, L\right]\end{cases}
$$

where $x_{i}^{n}$ is the unique point in $[0, L]$ such that the derivative of $u_{i-1}^{n}$ evaluated in $x_{i}^{n}$ is equal to $\left\{V_{i}^{n}-\right.$ $\left.u_{i-1}^{n}\left(x_{i}^{n}\right)\right\} /\left(L-x_{i}^{n}\right)[9]$.

In case $V_{i}^{n}>V_{i-1}^{n}$, we proceed analogously to the initial case $i=0$.

Suppose that there exist two solutions $w_{i}^{n} \neq u_{i}^{n}$. As shown in the case $i=0$, we can assume $w_{i}^{n} \geq u_{i}^{n}$. Let $y_{i}^{n} \leq x_{i}^{n}$ be the points defined by

$$
\begin{aligned}
& y_{i}^{n}:=\sup \left\{x \in[0, L]: w_{i}^{n}(x)=u_{i-1}^{n}(x)\right\} \\
& x_{i}^{n}:=\max \left\{\sup \left\{x \in[0, L]: u_{i}^{n}(x)=0\right\}, y_{i}^{n}\right\}
\end{aligned}
$$

From the continuity of the derivative of $u_{i}^{n}$ and of $w_{i}^{n}$ (Lem. 2, point II) and the fact that $u_{i}^{n} \geq u_{i-1}^{n}$, it follows $u_{i-1}^{n}\left(y_{i}^{n}\right)=w_{i}^{n}\left(y_{i}^{n}\right)=u_{i}^{n}\left(x_{i}^{n}\right)$,

$$
\frac{\mathrm{d} u_{i-1}^{n}}{\mathrm{~d} x}\left(y_{i}^{n}\right)=\frac{\mathrm{d} w_{i}^{n}}{\mathrm{~d} x}\left(y_{i}^{n}\right)=\frac{\mathrm{d} u_{i}^{n}}{\mathrm{~d} x}\left(x_{i}^{n}\right)
$$

and, using the convexity of $w_{i}^{n}$,

$$
\frac{\mathrm{d} u_{i}^{n}}{\mathrm{~d} x}\left(x_{i}^{n}\right) \leq \frac{\mathrm{d} w_{i}^{n}}{\mathrm{~d} x}\left(x_{i}^{n}\right)
$$

For $u_{i}^{n}$, we write the Euler-Lagrange condition in the inequality form, i.e.

$$
0 \leq N \int_{x_{i}^{n}}^{L} \frac{\mathrm{d} u_{i}^{n}}{\mathrm{~d} x} \frac{\mathrm{d} v_{0}}{\mathrm{~d} x}+\int_{x_{i}^{n}}^{L} \phi_{-}^{\prime}\left(\delta_{i-1}^{n}+\left[u_{i}^{n}-u_{i-1}^{n}\right]^{+}\right) v_{0}
$$

for any $v_{0} \in \mathbf{W}_{0}^{1,2}\left(x_{i}^{n}, L\right), v_{0} \geq 0$, meanwhile, for $w_{i}^{n}$, we write the Euler-Lagrange condition in the equation form, i.e.

$$
0=N \int_{y_{i}^{n}}^{L} \frac{\mathrm{d} w_{i}^{n}}{\mathrm{~d} x} \frac{\mathrm{d} v_{0}}{\mathrm{~d} x}+\int_{y_{i}^{n}}^{L} \phi_{-}^{\prime}\left(\delta_{i-1}^{n}+\left[w_{i}^{n}-u_{i-1}^{n}\right]^{+}\right) v_{0}
$$


for any $v_{0} \in \mathbf{W}_{0}^{1,2}\left(y_{i}^{n}, L\right)$. Furthermore, using the regularity of $u_{i}^{n}$ and $w_{i}^{n}$, we obtain

$$
\begin{aligned}
& N \frac{\mathrm{d}^{2} u_{i}^{n}}{\mathrm{~d} x^{2}}(x) \leq \phi_{-}^{\prime}\left(\delta_{i-1}^{n}(x)+\left[u_{i}^{n}(x)-u_{i-1}^{n}(x)\right]^{+}\right), \quad \text { for a.e. } x \in\left[x_{i}^{n}, L\right], \\
& N \frac{\mathrm{d}^{2} w_{i}^{n}}{\mathrm{~d} x^{2}}(x)=\phi_{-}^{\prime}\left(\delta_{i-1}^{n}(x)+\left[w_{i}^{n}(x)-u_{i-1}^{n}(x)\right]^{+}\right), \quad \text { for a.e. } x \in\left[y_{i}^{n}, L\right] .
\end{aligned}
$$

Recalling that $w_{i}^{n}$ and $u_{i}^{n}$ are strictly increasing respectively in $\left[y_{i}^{n}, L\right]$ and $\left[x_{i}^{n}, L\right]$ (point IV, Lem. 2), their inverse functions $w_{i}^{n,-1}$ and $u_{i}^{n,-1}$ are well defined in $\left[u_{i}^{n}\left(x_{i}^{n}\right), V_{i}^{n}\right]$.

In order to proceed with the same method as the one we used in the proof of uniqueness in the initial case $i=0$, we need that, for any fixed $z$, the function $\delta_{i-1}^{n}+\left[z-u_{i-1}^{n}\right]^{+}$is non-decreasing in $[0, L]$.

To prove that, fix $z$ in $\left[u_{i}^{n}\left(x_{i}^{n}\right), V_{i}^{n}\right]$ and let $x_{z}$ in $[0, L]$ be such that $z \leq u_{i-1}^{n}$ in $\left[x_{z}, L\right]$ (such a point exists since $u_{i-1}^{n}$ is a non-decreasing function in $\left.[0, L]\right)$. Observe that

$$
\frac{\mathrm{d} \delta_{i-1}^{n}}{\mathrm{~d} x} \geq \frac{\mathrm{d} u_{i-1}^{n}}{\mathrm{~d} x} \geq 0^{2} .
$$

Hence, $\delta_{i-1}^{n}+z-u_{i-1}^{n}$ and $\delta_{i-1}^{n}$ are non-decreasing respectively in $\left[0, x_{z}\right]$ and $\left[x_{z}, L\right]$. By the continuity of $\delta_{i-1}^{n}+\left[z-u_{i-1}^{n}\right]^{+}$, we conclude that $\delta_{i-1}^{n}+\left[z-u_{i-1}^{n}\right]^{+}$is non-decreasing in $[0, L]$.

Using the fact above, that $w_{i}^{n,-1} \leq u_{i}^{n,-1}$ and that $\phi^{\prime}$ is non-increasing, we have

$$
\begin{aligned}
N \frac{\mathrm{d}^{2} u_{i}^{n}}{\mathrm{~d} x^{2}}\left(u_{i}^{n,-1}(z)\right) & \leq \phi^{\prime}\left(\delta_{i-1}^{n}\left(u_{i}^{n,-1}(z)\right)+\left[z-u_{i-1}^{n}\left(u_{i}^{n,-1}(z)\right)\right]^{+}\right) \\
& \leq \phi^{\prime}\left(\delta_{i-1}^{n}\left(w_{i}^{n,-1}(z)\right)+\left[z-u_{i-1}^{n}\left(w_{i}^{n,-1}(z)\right)\right]^{+}\right)=N \frac{\mathrm{d}^{2} w_{i}^{n}}{\mathrm{~d} x^{2}}\left(w_{i}^{n,-1}(z)\right),
\end{aligned}
$$

for any $z$ in $\left[u_{i}^{n}\left(x_{i}^{n}\right), V_{i}^{n}\right]$. That gives rise to a contradiction.

Indeed, fix $x$ in $\left(x_{i}^{n}, L\right]$. By integrating over $\left[u_{i}^{n}\left(x_{i}^{n}\right), u_{i}^{n}(x)\right]$ the inequality above and the changes of variable $z=u_{i}^{n}(y)$ and $z=w_{i}^{n}(y)$, we obtain

$$
\begin{aligned}
& \frac{N}{2}\left\{\left|\frac{\mathrm{d} u_{i}^{n}}{\mathrm{~d} x}(x)\right|^{2}-\left|\frac{\mathrm{d} u_{i}^{n}}{\mathrm{~d} x}\left(x_{i}^{n}\right)\right|^{2}\right\}=N \int_{x_{i}^{n}}^{x} \frac{\mathrm{d}^{2} u_{i}^{n}}{\mathrm{~d} x^{2}}(y) \frac{\mathrm{d} u_{i}^{n}}{\mathrm{~d} x}(y) \mathrm{d} y=N \int_{u_{i}^{n}\left(x_{i}^{n}\right)}^{u_{i}^{n}(x)} \frac{\mathrm{d}^{2} u_{i}^{n}}{\mathrm{~d} x^{2}}\left(u_{i}^{n,-1}(z)\right) \mathrm{d} z \\
& \leq N \int_{u_{i}^{n}\left(x_{i}^{n}\right)}^{u^{n}(x)} \frac{\mathrm{d}^{2} w_{i}^{n}}{\mathrm{~d} x^{2}}\left(w_{i}^{n,-1}(z)\right) \mathrm{d} z=N \int_{x_{i}^{n}}^{w_{i}^{n,-1}\left(u_{i}^{n}(x)\right)} \frac{\mathrm{d}^{2} w_{i}^{n}}{\mathrm{~d} x^{2}}(y) \frac{\mathrm{d} w_{i}^{n}}{\mathrm{~d} x}(y) \mathrm{d} y \\
& \quad=\frac{N}{2}\left\{\left|\frac{\mathrm{d} w_{i}^{n}}{\mathrm{~d} x}\left(w_{i}^{n,-1}\left(u_{i}^{n}(x)\right)\right)\right|^{2}-\left|\frac{\mathrm{d} w_{i}^{n}}{\mathrm{~d} x}\left(x_{i}^{n}\right)\right|^{2}\right\}
\end{aligned}
$$

(we recall that $w_{i}^{n}\left(x_{i}^{n}\right)=u_{i}^{n}\left(x_{i}^{n}\right), x_{i}^{n}=w_{i}^{n,-1}\left(u_{i}^{n}\left(x_{i}^{n}\right)\right)$ ).

\footnotetext{
${ }^{2}$ In fact, for $j=0, \delta_{0}^{n}=u_{0}^{n}$; hence, $\frac{\mathrm{d} \delta_{0}^{n}}{\mathrm{~d} x}=\frac{\mathrm{d} u_{0}^{n}}{\mathrm{~d} x}$. For $j \geq 1$, assuming that $\frac{\mathrm{d} \delta_{j-1}^{n}}{\mathrm{~d} x} \geq \frac{\mathrm{d} u_{j-1}^{n}}{\mathrm{~d} x}$, we obtain

$$
\frac{\mathrm{d} \delta_{j}^{n}}{\mathrm{~d} x}= \begin{cases}\frac{\mathrm{d} \delta_{j-1}^{n}}{\mathrm{~d} x}+\frac{\mathrm{d} u_{j}^{n}}{\mathrm{~d} x}-\frac{\mathrm{d} u_{j-1}^{n}}{\mathrm{~d} x} \geq \frac{\mathrm{d} u_{j}^{n}}{\mathrm{~d} x}, & u_{j}^{n}>u_{j-1}^{n} \\ \frac{\mathrm{d} \delta_{j-1}^{n}}{\mathrm{~d} x} \geq \frac{\mathrm{d} u_{j-1}^{n}}{\mathrm{~d} x}=\frac{\mathrm{d} u_{j}^{n}}{\mathrm{~d} x}, & u_{j}^{n}=u_{j-1}^{n}\end{cases}
$$

where we used the fact that $u_{j}^{n} \geq u_{j-1}^{n}$ and that $\left\{u_{j}^{n}=u_{j-1}^{n}\right\}$ is an interval (those are consequences of point I, Cor. 1)
} 
Observe that $u_{i}^{n}(x) \leq w_{i}^{n}(x), w_{i}^{n,-1}\left(u_{i}^{n}(x)\right) \leq x$. Then, by the inequality $\frac{\mathrm{d} u_{i}^{n}}{\mathrm{~d} x}\left(x_{i}^{n}\right) \leq \frac{\mathrm{d} w_{i}^{n}}{\mathrm{~d} x}\left(x_{i}^{n}\right)$ and the convexity of $w_{i}^{n}$,

$$
\frac{\mathrm{d} u_{i}^{n}}{\mathrm{~d} x}(x) \leq \frac{\mathrm{d} w_{i}^{n}}{\mathrm{~d} x}\left(w_{i}^{n,-1}\left(u_{i}^{n}(x)\right)\right) \leq \frac{\mathrm{d} w_{i}^{n}}{\mathrm{~d} x}(x) .
$$

Since $u_{i}^{n} \neq w_{i}^{n}$, there exists at least a point $x^{n}$ in $\left(x_{i}^{n}, L\right]$ whit $u_{i}^{n}\left(x^{n}\right)<w_{i}^{n}\left(x^{n}\right)$. But from the above inequality,

$$
V_{i}^{n}-u_{i}^{n}\left(x^{n}\right)=\int_{x^{n}}^{L} \frac{\mathrm{d} u_{i}^{n}}{\mathrm{~d} x} \leq \int_{x^{n}}^{L} \frac{\mathrm{d} w_{i}^{n}}{\mathrm{~d} x}=V_{i}^{n}-w_{i}^{n}\left(x^{n}\right),
$$

that implies, $w_{i}^{n}\left(x^{n}\right) \leq u_{i}^{n}\left(x^{n}\right)$, a contradiction. Therefore, we conclude that there cannot exists two different solutions to $\left(\mathbf{P}_{i}^{n}\right)$.

We sum up below some important properties of the solutions $u_{i}^{n}$ that follow directly from uniqueness. Even if their proofs are partially contained in the proof of Theorem 1, we rewrite them for reader convenience.

Corollary 1. The $u_{i}^{n}$ enjoy the following properties:

I. if $u_{i}^{n}(x)=u_{i-1}^{n}(x)$ in $x \in[0, L]$, then $u_{i}^{n}=u_{i-1}^{n}$ in $[0, x]$;

II. if $V_{i}^{n} \geq V_{i-1}^{n}$, then $u_{i}^{n} \geq u_{i-1}^{n}$;

III. if $V_{i}^{n} \leq V_{i-1}^{n}$, then $u_{i}^{n} \leq u_{i-1}^{n}$;

IV. if $V_{i}^{n}>V_{i-1}^{n} \geq V_{i-2}^{n}$, then $x_{i}^{n} \leq x_{i-1}^{n}$, where $x_{i}^{n}:=\sup \left\{x \in[0, L]: u_{i}^{n}(x)=u_{i-1}^{n}\right\}$ and $x_{i-1}^{n}:=\sup \{x \in$ $\left.[0, L]: u_{i-1}^{n}(x)=u_{i-2}^{n}\right\}$

V. if $V_{i}^{n}<V_{i-1}^{n} \leq V_{i-2}^{n}$, then $x_{i}^{n} \leq x_{i-1}^{n}$.

Proof of I. Consider the competitors given by $\bar{u}_{i}^{n}:=u_{i-1}^{n} \chi_{[0, x]}+u_{i}^{n} \chi_{(x, L]}$ and $\bar{u}_{i-1}^{n}:=u_{i}^{n} \chi_{[0, x]}+u_{i-1}^{n} \chi_{(x, L]}$. Then, observing that $\bar{u}_{i}^{n}=u_{i}^{n}$ and $\bar{u}_{i-1}^{n}=u_{i-1}^{n}$ on $(x, L]$,

$$
\begin{aligned}
& 0 \leq \mathcal{I}_{i}^{n}\left(\bar{u}_{i}^{n}\right)-\mathcal{I}_{i}^{n}\left(u_{i}^{n}\right)=\left.\mathcal{I}_{i}^{n}\right|_{[0, x]}\left(u_{i-1}^{n}\right)-\left.\mathcal{I}_{i}^{n}\right|_{[0, x]}\left(u_{i}^{n}\right) \\
& 0 \leq \mathcal{I}_{i-1}^{n}\left(\bar{u}_{i-1}^{n}\right)-\mathcal{I}_{i-1}^{n}\left(u_{i-1}^{n}\right)=\left.\mathcal{I}_{i-1}^{n}\right|_{[0, x]}\left(u_{i}^{n}\right)-\left.\mathcal{I}_{i-1}^{n}\right|_{[0, x]}\left(u_{i-1}^{n}\right)
\end{aligned}
$$

$\left(\left.\mathcal{I}_{i}^{n}\right|_{[0, x]}\right.$ denotes the functional $\mathcal{I}_{i}^{n}$ where the integrals in there are taken on $\left.[0, x]\right)$. It follows directly from the sub-additivity of $\phi$ and of $[\cdot]^{+}$that

$$
\begin{aligned}
\left.\mathcal{I}_{i-1}^{n}\right|_{[0, x]}\left(u_{i}^{n}\right)=\frac{N}{2} \int_{0}^{x}\left|\frac{\mathrm{d} u_{i}^{n}}{\mathrm{~d} x}\right|^{2}+ & \int_{0}^{x} \phi\left(\delta_{i-2}^{n}+\left[u_{i}^{n}-u_{i-2}^{n}\right]^{+}\right) \\
& \leq \frac{N}{2} \int_{0}^{x}\left|\frac{\mathrm{d} u_{i}^{n}}{\mathrm{~d} x}\right|^{2}+\int_{0}^{x} \phi\left(\delta_{i-2}^{n}+\left[u_{i}^{n}-u_{i-1}^{n}\right]+\left[u_{i-1}^{n}-u_{i-2}^{n}\right]^{+}\right) \leq\left.\mathcal{I}_{i}^{n}\right|_{[0, x]}\left(u_{i}^{n}\right),
\end{aligned}
$$

and, from the definition of $\delta_{i}^{n}$, that

$$
\begin{aligned}
\left.\mathcal{I}_{i-1}^{n}\right|_{[0, x]}\left(u_{i-1}^{n}\right)=\frac{N}{2} \int_{0}^{x}\left|\frac{\mathrm{d} u_{i-1}^{n}}{\mathrm{~d} x}\right|^{2}+\int_{0}^{x} \phi\left(\delta_{i-1}^{n}\right) & =\frac{N}{2} \int_{0}^{x}\left|\frac{\mathrm{d} u_{i-1}^{n}}{\mathrm{~d} x}\right|^{2}+\int_{0}^{x} \phi\left(\delta_{i-1}^{n}+\left[u_{i-1}^{n}-u_{i-1}^{n}\right]^{+}\right)=\left.\mathcal{I}_{i}^{n}\right|_{[0, x]}\left(u_{i-1}^{n}\right) .
\end{aligned}
$$

Therefore,

$$
\left.\mathcal{I}_{i-1}^{n}\right|_{[0, x]}\left(u_{i}^{n}\right)-\left.\mathcal{I}_{i-1}^{n}\right|_{[0, x]}\left(u_{i-1}^{n}\right) \leq\left.\mathcal{I}_{i}^{n}\right|_{[0, x]}\left(u_{i}^{n}\right)-\left.\mathcal{I}_{i}^{n}\right|_{[0, x]}\left(u_{i-1}^{n}\right) \leq 0,
$$

and, hence, $\mathcal{I}_{i-1}^{n}\left(\bar{u}_{i-1}^{n}\right)=\mathcal{I}_{i-1}^{n}\left(u_{i-1}^{n}\right)$. It follows, by the uniqueness of $u_{i-1}^{n}, \bar{u}_{i-1}^{n}=u_{i-1}^{n}$ and, by the definition of $\bar{u}_{i-1}^{n}, u_{i}^{n}=u_{i-1}^{n}$ in $[0, x]$. 
Proof of II. Since $V_{i}^{n} \geq V_{i-1}^{n}$, then $u_{i}^{n} \geq u_{i-1}^{n}$ in a left neighbourhood of $L$. By point I, if there exists $x$ in $[0, L)$ such that $u_{i}^{n}(x)=u_{i-1}^{n}(x)$ in $x \in[0, L]$, then $u_{i}^{n}=u_{i-1}^{n}$ in $[0, x]$. Hence, $u_{i}^{n} \geq u_{i-1}^{n}$ in $[0, L]$.

Proof of III. Since $V_{i}^{n} \leq V_{i-1}^{n}$, then $u_{i}^{n} \leq u_{i-1}^{n}$ in a left neighbourhood of $L$. By point I, if there exists $x$ in $[0, L)$ such that $u_{i}^{n}(x)=u_{i-1}^{n}(x)$ in $x \in[0, L]$, then $u_{i}^{n}=u_{i-1}^{n}$ in $[0, x]$. Hence, $u_{i}^{n} \leq u_{i-1}^{n}$ in $[0, L]$.

Proof of IV. Suppose $x_{i}^{n}>x_{i-1}^{n}$. By point II, $u_{i}^{n} \geq u_{i-1}^{n}$.

Integrating the Euler-Lagrange equation (in the classical form) on the interval $\left[x_{i}^{n}, x\right]$, we obtain

$$
N \frac{\mathrm{d} u_{i}^{n}}{\mathrm{~d} x}(x)-N \frac{\mathrm{d} u_{i}^{n}}{\mathrm{~d} x}\left(x_{i}^{n}\right)=\int_{x_{i}^{n}}^{x} \phi_{-}^{\prime}\left(\delta_{i}^{n}(x)\right) \leq \int_{x_{i}^{n}}^{x} \phi_{-}^{\prime}\left(\delta_{i-1}^{n}(x)\right)=N \frac{\mathrm{d} u_{i-1}^{n}}{\mathrm{~d} x}(x)-N \frac{\mathrm{d} u_{i-1}^{n}}{\mathrm{~d} x}\left(x_{i}^{n}\right) .
$$

Since, by the continuity of the derivative of the minimizers, $\frac{\mathrm{d} u_{i}^{n}}{\mathrm{~d} x}\left(x_{i}^{n}\right)=\frac{\mathrm{d} u_{i-1}^{n}}{\mathrm{~d} x}\left(x_{i}^{n}\right)$, we infer that $\frac{\mathrm{d} u_{i}^{n}}{\mathrm{~d} x} \leq \frac{\mathrm{d} u_{i-1}^{n}}{\mathrm{~d} x}$ in $\left[x_{i}^{n}, L\right]$ and, hence,

$$
u_{i}^{n}(x)-u_{i}^{n}\left(x_{i}^{n}\right)=\int_{x_{i}^{n}}^{x} \frac{\mathrm{d} u_{i}^{n}}{\mathrm{~d} x} \leq \int_{x_{i}^{n}}^{x} \frac{\mathrm{d} u_{i-1}^{n}}{\mathrm{~d} x}=u_{i-1}^{n}(x)-u_{i-1}^{n}\left(x_{i}^{n}\right) .
$$

From the definition of $x_{i}^{n}$, it follows that $u_{i}^{n} \leq u_{i-1}^{n}$ in $\left[x_{i}^{n}, L\right]$. That contradicts the assumption $V_{i}^{n}>V_{i-1}^{n}$.

Proof of V. Recall that, in case $V_{i}^{n} \leq V_{i-1}^{n}$, problem $\left(\mathbf{P}_{i}^{n}\right)$ is equivalent to minimize

$$
\frac{N}{2} \int_{0}^{L}\left|\frac{\mathrm{d} u}{\mathrm{~d} x}\right|^{2}+\int_{0}^{L} \phi\left(\delta_{i-1}^{n}\right)=\frac{N}{2} \int_{0}^{L}\left|\frac{\mathrm{d} u}{\mathrm{~d} x}\right|^{2}+\text { const. }
$$

on the set of functions $u$ of $\mathbf{W}^{1,2}(0, L)$ satisfying $u(0)=0, u(L)=V_{i}^{n}$ and $u \leq u_{i-1}^{n}$. That is an obstacle problem with convex obstruction $u_{i-1}^{n}$ (Lem. 2, point III). The unique solution is

$$
u_{i}^{n}(x)= \begin{cases}u_{i-1}^{n}(x), & x \in\left[0, x_{i}^{n}\right] \\ \left(x-x_{i}^{n}\right)\left\{V_{i}^{n}-u_{i-1}^{n}\left(x_{i}^{n}\right)\right\} /\left(L-x_{i}^{n}\right)+u_{i-1}^{n}\left(x_{i}^{n}\right), & x \in\left(x_{i}^{n}, L\right]\end{cases}
$$

where $x_{i}^{n}$ is the unique point in $[0, L]$ such that the derivative of $u_{i-1}^{n}$ evaluated in $x_{i}^{n}$ is equal to $\left\{V_{i}^{n}-\right.$ $\left.u_{i-1}^{n}\left(x_{i}^{n}\right)\right\} /\left(L-x_{i}^{n}\right)[9]$.

Suppose $x_{i}^{n}>x_{i-1}^{n}$. Since the problem $\left(\mathbf{P}_{i-1}^{n}\right)$ is an obstacle problem to, we have that $u_{i-1}^{n}$ is a straight line in $\left[x_{i-1}^{n}, L\right]$. From the continuity of the derivative of the minimizers, it follows

$$
\frac{V_{i}^{n}-u_{i-1}^{n}\left(x_{i}^{n}\right)}{L-x_{i}^{n}}=\frac{V_{i-1}^{n}-u_{i-2}^{n}\left(x_{i-1}^{n}\right)}{L-x_{i-1}^{n}}=\frac{V_{i-1}^{n}-u_{i-1}^{n}\left(x_{i}^{n}\right)}{L-x_{i}^{n}},
$$

which implies $V_{i}^{n}=V_{i-1}^{n}$. That contradicts the assumption $V_{i}^{n}<V_{i-1}^{n}$.

We conclude this section with two important properties of our formulation. The time discrete evolution is independent on the discretization and the debonded zone grows for each cycle.

This is proved in the lemma below.

Lemma 3. Let $\mathcal{P}^{n}$ be a partition of $[0, T]$ that contains $\{0, T\}$ and the points of the boundary of the open set $\{t \in(0, T): \dot{V}$ is continuous at $t, \dot{V}(t)>0\}=\cup_{r=1}^{h}\left(a_{r}, b_{r}\right)$. Then:

I. If $\mathcal{P}^{m}=\left\{0=t_{0}^{m}<\cdots<t_{j}^{m}<\cdots<t_{q(m)}^{m}=T\right\}$ is a refinement of $\mathcal{P}^{n}$, i.e. $\mathcal{P}^{n} \subset \mathcal{P}^{m}$, then, for any $t_{i}^{n} \in \mathcal{P}^{n}, u_{i}^{n}=u_{j(i)}^{m}$ whenever $t_{j(i)}^{m}=t_{i}^{n}$;

II. The debonded zone grows for cycle, i.e. if $V\left(b_{r+1}\right) \geq V\left(b_{r}\right)>0$ for any $r=1, \ldots, h$, then $x_{b_{r+1}} \leq x_{b_{r}}$, where $x_{b_{r}}:=\sup \left\{x \in[0, L]: u_{b_{r}}(x)=0\right\}$ and $u_{b_{r}}$ denotes the solution with boundary value $V\left(b_{r}\right)$. 
Proof of I. This is a straightforward consequence of the uniqueness of solutions (Th. 1).

We prove the result for a refinement $\mathcal{P}^{m}$ with the property that any two consecutive points $t_{i-1}^{n}<t_{i}^{n}$ of $\mathcal{P}^{n}$ are separated by at most one point $t_{j}^{m}$ of $\mathcal{P}^{m}$. The case of a general refinement can be obtained by writing it as a finite union of partial refinements with the property above, and applying recursively the lemma at each partial union.

We argue by induction on $i$. Assume that $u_{\hat{1}}^{n}=u_{\hat{\jmath}}^{m}$ and $\delta_{\hat{1}}^{n}=\delta_{\hat{\jmath}}^{m}$ whenever $\hat{\imath}<i$ and $t_{\hat{1}}^{n}=t_{\hat{\jmath}}^{m}$. Assume also that $t_{i-1}^{n}=t_{j-1}^{m}<t_{j}^{m}<t_{j+1}^{m}=t_{i}^{n}$. We want to prove that $u_{j+1}^{m}=u_{i}^{n}$ and $\delta_{j+1}^{m}=\delta_{i}^{n}$. Since $\mathcal{P}^{n}$ contains the boundary of $\{t \in[0, T]: \dot{V}$ is continuous at $t, \dot{V}(t)>0\}$, either $V_{i}^{n}>V_{j}^{m}>V_{i-1}^{n}$ or $V_{i}^{n} \leq V_{j}^{m} \leq V_{i-1}^{n}$.

Consider the first case. Since $u_{i-1}^{n}=u_{j-1}^{m}$ and $\delta_{i-1}^{n}=\delta_{j-1}^{m}$ by the inductive assumption, we have $\mathcal{I}_{j}^{m}=\mathcal{I}_{i}^{n}$. By Corollary 1 this implies $u_{i}^{n} \geq u_{i-1}^{n}$ and $u_{j}^{m} \geq u_{j-1}^{m}=u_{i-1}^{n}$. Let us prove that we have also that $u_{i}^{n} \geq u_{j}^{m}$. Indeed, if this is false, since $u_{i}^{n} \geq u_{j}^{m}$ in a left neighbourhood of $L$, there is a point $x$ in $[0, L]$ such that $u_{i}^{n}(x)=u_{j}^{m}(x)$ and $u_{i}^{n} \neq u_{j}^{m}$ in $[0, x]$. As the functionals $\left.\mathcal{I}_{i}^{n}\right|_{[0, x]}$ and $\left.\mathcal{I}_{j}^{m}\right|_{[0, x]}$ coincide by the inductive hypothesis, there would be two minimizers to the functional $\left.\mathcal{I}_{i}^{n}\right|_{[0, x]}$ in the class of functions $u$ with boundary values $u(0)=0$ and $u(x)=u_{i}^{n}(x)$; that contradicts Theorem 1 in the interval $[0, x]$.

Now, consider the problem $\left(\mathcal{P}_{j+1}^{m}\right)$. By the fact that $u_{i}^{n} \geq u_{j}^{m} \geq u_{i-1}^{n}=u_{j-1}^{m}$ and $u_{j+1}^{m} \geq u_{j}^{m}$, taking into account the expressions of $\delta_{i-1}^{n}$ and $\delta_{j}^{m}$, we obtain

$$
\mathcal{I}_{j+1}^{m}\left(u_{i}^{n}\right)=\mathcal{I}_{i}^{n}\left(u_{i}^{n}\right) \leq \mathcal{I}_{i}^{n}\left(u_{j+1}^{m}\right)=\mathcal{I}_{j+1}^{m}\left(u_{j+1}^{m}\right) .
$$

Hence, by Theorem 1, $u_{j+1}^{m}=u_{i}^{n}$. As $u_{j+1}^{m}=u_{i}^{n} \geq u_{j}^{m} \geq u_{i-1}^{n}=u_{j-1}^{m}$, from $\delta_{i-1}^{n}=\delta_{j-1}^{m}$ we obtain also $\delta_{j+1}^{m}=\delta_{i}^{n}$.

In the second case, we consider two subcases. In case one of the two inequalities is an equality the result follows directly from Theorem 1 because $u_{j}^{m}$ would be equal to $u_{i}^{n}$ or to $u_{i-1}^{n}$. The proof of the remaining subcase $V_{i}^{n}<V_{j}^{m}<V_{i-1}^{n}$ proceeds as the proof of the first case.

Proof of II. By point I, we can assume $\mathcal{P}^{n}=\left\{0=t_{0}^{n}<\cdots<t_{i}^{n}<\cdots<t_{p(n)}^{n}=T\right\}=\left\{0, a_{1}, b_{1}, \ldots, a_{h}, b_{h}, T\right\}$. To simplify the notation, we set $u_{a_{r}}=u_{i}^{n}$ for $t_{i}^{n}=a_{r}$ and $u_{b_{r}}=u_{j}^{n}$ for $t_{j}^{n}=b_{r}$.

Suppose $V\left(a_{r+1}\right)>0$. By point III of Corollary $1, u_{b_{r}} \geq u_{a_{r+1}}$. Hence, as we have already observed in the proof of Theorem 1, there exists a point $x \in[0, L]$ such that $u_{a_{r+1}}=u_{b_{r}}$ in $[0, x]$ and $u_{a_{r+1}}$ is a straight line in $[x, L]$. Since $u_{b_{r}}(x)>0$ and $u_{b_{r+1}} \geq u_{a_{r+1}}$, we conclude that $x_{b_{r+1}} \leq x_{b_{r}}$.

Suppose $V\left(a_{r+1}\right)=0$. Let $r_{0}$ be the greatest element in $\{1, \ldots, r\}$ such that $V\left(a_{r_{0}}\right)=0$, and $\operatorname{set} \bar{r}:=\min \{j:$ $\left.r_{0} \leq j \leq r, x_{b_{j}}=\min \left\{x_{b_{i}}: r_{0} \leq i \leq r\right\}\right\}$.

We claim that $u_{b_{\bar{r}}}$ satisfy the Euler-Lagrange equation in $\left[x_{b_{\bar{r}}}, L\right]$. In fact, if $\bar{r}=r_{0}$, then $u_{b_{r_{0}}}>0=u_{a_{r_{0}}}$ on $\left[x_{b_{r_{0}}}, L\right]$, since $V\left(b_{r_{0}}\right)>0=V\left(a_{r_{0}}\right)$. Otherwise, $x_{b_{\bar{r}}}<x_{b_{\bar{r}-1}}$ (by the minimal property of $x_{b_{\bar{r}}}$ and $\bar{r}$ ) and $x_{a_{\bar{r}}}=x_{b_{\bar{r}-1}}$ (since $V\left(a_{\bar{r}}\right)>0$ by definition of $\left.r_{0}\right)$. That implies $u_{b_{\bar{r}}}>u_{a_{\bar{r}}}$ on $\left[x_{b_{\bar{r}}}, L\right]$. Therefore, the Euler-Lagrange equation (2) for $u_{b_{\bar{r}}}$ is

$$
N \frac{\mathrm{d}^{2} u_{b_{\bar{\tau}}}}{\mathrm{d} x^{2}}(x)=\phi_{-}^{\prime}\left(\delta_{b_{\bar{r}}}(x)\right), \text { for any } x \in\left[x_{b_{\bar{r}}}, L\right] .
$$

Since $u_{a_{r+1}}$ is identically 0 , the Euler-Lagrange equation for $u_{b_{r+1}}$ is

$$
N \frac{\mathrm{d}^{2} u_{b_{r+1}}}{\mathrm{~d} x^{2}}(x)=\phi_{-}^{\prime}\left(\delta_{a_{r+1}}(x)+u_{b_{r+1}}(x)\right), \quad \text { for any } x \in\left[x_{b_{r+1}}, L\right] .
$$

Suppose that $x_{b_{r+1}}>x_{b_{\bar{r}}}$. Then, since $\delta_{a_{r+1}}+u_{b_{r+1}} \geq \delta_{a_{r+1}} \geq \delta_{b_{\bar{r}}}$ and $\phi_{-}^{\prime}$ is non-increasing, we have, for any $x \in\left(x_{b_{r+1}}, L\right]$,

$$
\frac{\mathrm{d} u_{b_{r+1}}}{\mathrm{~d} x}(x)=\frac{1}{N} \int_{x_{b_{r+1}}}^{x} \phi_{-}^{\prime}\left(\delta_{a_{r+1}}+u_{b_{r+1}}\right) \leq \frac{1}{N} \int_{x_{b_{r+1}}}^{x} \phi_{-}^{\prime}\left(\delta_{b_{\bar{r}}}\right) \leq \frac{\mathrm{d} u_{b_{\bar{r}}}}{\mathrm{~d} x}(x) .
$$

If follows that $V\left(b_{\bar{r}}\right) \leq V\left(b_{r+1}\right) \leq V\left(b_{\bar{r}}\right)-u_{b_{\bar{r}}}\left(x_{b_{r+1}}\right)$, and, hence, $u_{b_{\bar{r}}}\left(x_{b_{r+1}}\right) \leq 0$. That implies $x_{b_{r+1}} \leq x_{b_{\bar{r}}}$, a contradiction. 
By definition of $\bar{r}, x_{b_{\bar{r}}} \leq x_{b_{r}}$ and we conclude that $x_{b_{r+1}} \leq x_{b_{r}}$.

\section{A PRIORI Estimates}

To perform the limit as the time step of the discretization $\mathcal{P}^{n}$ goes to 0 , as $n$ goes to $\infty$, we use compactness properties. In order to do that, we need $u_{i}^{n}$ to be a priori bounded in some appropriate norm. This is the object of this section.

We extend any $u_{i}^{n}$ to a piecewise constant map $u^{n}$ defined in $[0, T]$ : for any $n$ in $\mathbb{N}, u^{n}$ from $[0, T]$ to $\mathbf{W}^{1,2}(0, L)$ is given by

where $\left\{0=t_{0}^{n}<t_{1}^{n}<\cdots<t_{p(n)}^{n}=T\right\}=\mathcal{P}^{n}$.

$$
u^{n}(t):=\sum_{k=1}^{p(n)} u_{k-1}^{n} \chi_{\left[t_{k-1}^{n}, t_{k}^{n}\right)}(t)+u_{p(n)}^{n} \chi_{\{T\}}(t),
$$

(Notation: $\frac{\partial u}{\partial x}$ will denote as usual the partial derivative of $u=u(t, x)$ with respect to the space variable $x$, meanwhile $\dot{u}$ will denote the partial derivative of $u$ with respect to the time variable $t$. Besides, for any function $f$ from $[0, T]$ to $\mathbf{L}^{1}(0, L)$, we denotes by $\operatorname{Var}(f ;[0, T])$ the total variation on $[0, T]$ of the function $f$ in the $\mathbf{L}^{1}(0, L)$ norm.)

Theorem 2. The sequence $\left\{u^{n}\right\}_{n}$ satisfies the following estimates:
I. $\left\|\frac{\partial u^{n}}{\partial x}\right\|_{\mathbf{L}^{\infty}\left((0, T) ; \mathbf{W}^{1, \infty}(0, L)\right)} \leq \mathrm{c}_{1}$, for any $n$;
II. $\operatorname{Var}\left(\frac{\partial u^{n}}{\partial x} ;[0, T]\right) \leq \mathrm{c}_{2}$, for any $n$.

Proof of I. Recalling inequality (3) in point II of Lemma 2, we get immediately

$$
\operatorname{Lip}\left(\frac{\mathrm{d} u_{i}^{n}}{\mathrm{~d} x}\right) \leq \frac{\phi^{\prime}(0)}{N}
$$

for any $n$ in $\mathbb{N}$ and $i$ in $\{0,1, \ldots, p(n)\}$. Using the convexity of $u_{i}^{n},\left|\frac{\mathrm{d} u_{i}^{n}}{\mathrm{~d} x}(0)\right| \leq \frac{V_{i}^{n}}{L}$ and, hence, for any $n$

$$
\left\|\frac{\partial u^{n}}{\partial x}\right\|_{\mathbf{L}^{\infty}\left((0, T) ; \mathbf{W}^{1, \infty}(0, L)\right)} \leq \frac{\|V\|_{\mathbf{L}^{\infty}(0, T)}}{L}+\frac{\phi^{\prime}(0)}{N}=: \mathrm{c}_{1} .
$$

Proof of II. Fix $n$ in $\mathbb{N}$ and $i$ in $\{0,1, \ldots, p(n)\}$.

Consider the case $V_{i}^{n} \leq V_{i-1}^{n}$ (for $i=1$, we set $\left.V_{-1}:=0\right)$.

By Corollary 1, $u_{i}^{n} \leq u_{i-1}^{n}$. We have already noted in Theorem 1 that the problem $\left(\mathbf{P}_{i}^{n}\right)$ is equivalent to minimizing

$$
\frac{N}{2} \int_{0}^{L}\left|\frac{\mathrm{d} u}{\mathrm{~d} x}\right|^{2}+\int_{0}^{L} \phi\left(\delta_{i-1}^{n}\right)=\frac{N}{2} \int_{0}^{L}\left|\frac{\mathrm{d} u}{\mathrm{~d} x}\right|^{2}+\text { const. }
$$

on the set of functions $u$ of $\mathbf{W}^{1,2}(0, L)$ satisfying $u(0)=0, u(L)=V_{i}^{n}$ and $u \leq u_{i-1}^{n}$. That is an obstacle problem with convex obstruction $u_{i-1}^{n}$ (Lem. 2, point III). We recall that the unique solution is

$$
u_{i}^{n}(x)= \begin{cases}u_{i-1}^{n}(x), & x \in\left[0, x_{i}^{n}\right], \\ \left(x-x_{i}^{n}\right)\left\{V_{i}^{n}-u_{i-1}^{n}\left(x_{i}^{n}\right)\right\} /\left(L-x_{i}^{n}\right)+u_{i-1}^{n}\left(x_{i}^{n}\right), & x \in\left(x_{i}^{n}, L\right]\end{cases}
$$

where $x_{i}^{n}$ is the unique point in $[0, L]$ such that the derivative of $u_{i-1}^{n}$ evaluated in $x_{i}^{n}$ is equal to $\left\{V_{i}^{n}-\right.$ $\left.u_{i-1}^{n}\left(x_{i}^{n}\right)\right\} /\left(L-x_{i}^{n}\right)[9]$. 
Hence, we have that

$$
\left\|\frac{\mathrm{d} u_{i}^{n}}{\mathrm{~d} x}-\frac{\mathrm{d} u_{i-1}^{n}}{\mathrm{~d} x}\right\|_{\mathbf{L}^{1}(0, L)}=\int_{x_{i}^{n}}^{L}\left\{\frac{\mathrm{d} u_{i-1}^{n}}{\mathrm{~d} x}-\frac{V_{i}^{n}-u_{i-1}^{n}\left(x_{i}^{n}\right)}{L-x_{i}^{n}}\right\}=V_{i-1}^{n}-V_{i}^{n} .
$$

Now, consider the case $V_{i}^{n}>V_{i-1}^{n}$ (necessarily $i \geq 1$, because $V_{-1}^{n}=0$ ). We separate the proof in three subcases: $V_{i-1}^{n}<V_{i-2}^{n}, V_{i-1}^{n}>V_{i-2}^{n}$ and $V_{i-1}^{n}=V_{i-2}^{n}$.

Denote as usual by $x_{i}^{n}$ and $x_{i-1}^{n}$ the points

$$
\begin{aligned}
& x_{i}^{n}:=\sup \left\{x \in[0, L]: u_{i}^{n}(x)=u_{i-1}^{n}(x)\right\} \\
& x_{i-1}^{n}:=\sup \left\{x \in[0, L]: u_{i-1}^{n}(x)=u_{i-2}^{n}(x)\right\} .
\end{aligned}
$$

Suppose $V_{i-1}^{n}<V_{i-2}^{n}$.

Notice that, by point I of Corollary $1, u_{i}^{n}$ coincide with $u_{i-1}^{n}$ in the interval $\left[0, x_{i}^{n}\right]$ and, hence,

$$
\frac{\mathrm{d} u_{i}^{n}}{\mathrm{~d} x}(x)=\frac{\mathrm{d} u_{i-1}^{n}}{\mathrm{~d} x}(x), \quad \text { for any } x \in\left[0, x_{i}^{n}\right] .
$$

In case $x_{i}^{n} \geq x_{i-1}^{n}$, by point III of Corollary $1, u_{i-1}^{n}$ is a straight line in $\left(x_{i}^{n}, L\right]$ (recall the reduction to an obstacle problem that we have in this case). Therefore, by the equality $\frac{\mathrm{d} u_{i}^{n}}{\mathrm{~d} x}\left(x_{i}^{n}\right)=\frac{\mathrm{d} u_{i-1}^{n}}{\mathrm{~d} x}\left(x_{i}^{n}\right)$ (point II, Lem. 2) and the convexity of $u_{i}^{n}$, for any $x$ in $\left(x_{i}^{n}, L\right]$, we have

$$
\left|\frac{\mathrm{d} u_{i}^{n}}{\mathrm{~d} x}(x)-\frac{\mathrm{d} u_{i-1}^{n}}{\mathrm{~d} x}(x)\right|=\left|\frac{\mathrm{d} u_{i}^{n}}{\mathrm{~d} x}(x)-\frac{\mathrm{d} u_{i-1}^{n}}{\mathrm{~d} x}\left(x_{i}^{n}\right)\right| \leq \frac{\mathrm{d} u_{i}^{n}}{\mathrm{~d} x}(x)-\frac{\mathrm{d} u_{i-1}^{n}}{\mathrm{~d} x}\left(x_{i}^{n}\right) .
$$

Taking the integral over $\left(x_{i}^{n}, L\right]$, we obtain

$$
\int_{x_{i}^{n}}^{L}\left|\frac{\mathrm{d} u_{i}^{n}}{\mathrm{~d} x}-\frac{\mathrm{d} u_{i-1}^{n}}{\mathrm{~d} x}\right| \leq \int_{x_{i}^{n}}^{L} \frac{\mathrm{d} u_{i}^{n}}{\mathrm{~d} x}(x)-\frac{\mathrm{d} u_{i-1}^{n}}{\mathrm{~d} x}\left(x_{i}^{n}\right)=V_{i}^{n}-V_{i-1}^{n}-u_{i}^{n}\left(x_{i}^{n}\right)+u_{i-1}^{n}\left(x_{i}^{n}\right)=V_{i}^{n}-V_{i}^{n},
$$

by the definition of $x_{i}^{n}$.

In case $x_{i}^{n}<x_{i-1}^{n}$, fix $x$ in $\left(x_{i}^{n}, x_{i-1}^{n}\right]$. By the estimate in point I, we have

$$
\left|\frac{\mathrm{d} u_{i}^{n}}{\mathrm{~d} x}(x)-\frac{\mathrm{d} u_{i-1}^{n}}{\mathrm{~d} x}(x)\right| \leq\left|\frac{\mathrm{d} u_{i}^{n}}{\mathrm{~d} x}(x)-\frac{\mathrm{d} u_{i}^{n}}{\mathrm{~d} x}\left(x_{i}^{n}\right)\right|+\left|\frac{\mathrm{d} u_{i-1}^{n}}{\mathrm{~d} x}\left(x_{i}^{n}\right)-\frac{\mathrm{d} u_{i-1}^{n}}{\mathrm{~d} x}(x)\right| \leq 2 \frac{\phi^{\prime}(0)}{N}\left(x_{i-1}^{n}-x_{i}^{n}\right),
$$

that implies

$$
\int_{x_{i}^{n}}^{x_{i-1}^{n}}\left|\frac{\mathrm{d} u_{i-1}^{n}}{\mathrm{~d} x}-\frac{\mathrm{d} u_{i-1}^{n}}{\mathrm{~d} x}\right| \leq 2 \frac{\phi^{\prime}(0)}{N}\left(x_{i-1}^{n}-x_{i}^{n}\right)^{2} .
$$

Meanwhile, for any $x$ in $\left(x_{i-1}^{n}, L\right]$, by point III of Corollary $1, u_{i-1}^{n}$ is a straight line in $\left(x_{i-1}^{n}, L\right]$ (recall the reduction to an obstacle problem that we have in this case). Therefore, we have

$$
\begin{aligned}
\left|\frac{\mathrm{d} u_{i}^{n}}{\mathrm{~d} x}(x)-\frac{\mathrm{d} u_{i-1}^{n}}{\mathrm{~d} x}(x)\right| & =\left|\frac{\mathrm{d} u_{i}^{n}}{\mathrm{~d} x}(x)-\frac{\mathrm{d} u_{i-1}^{n}}{\mathrm{~d} x}\left(x_{i-1}^{n}\right)\right| \leq\left|\frac{\mathrm{d} u_{i}^{n}}{\mathrm{~d} x}(x)-\frac{\mathrm{d} u_{i}^{n}}{\mathrm{~d} x}\left(x_{i}^{n}\right)\right|+\left|\frac{\mathrm{d} u_{i-1}^{n}}{\mathrm{~d} x}\left(x_{i}^{n}\right)-\frac{\mathrm{d} u_{i-1}^{n}}{\mathrm{~d} x}\left(x_{i-1}^{n}\right)\right| \\
& \leq \frac{\mathrm{d} u_{i}^{n}}{\mathrm{~d} x}(x)-\frac{\mathrm{d} u_{i}^{n}}{\mathrm{~d} x}\left(x_{i}^{n}\right)+\frac{\phi^{\prime}(0)}{N}\left(x_{i-1}^{n}-x_{i}^{n}\right) \\
& \leq \frac{\mathrm{d} u_{i}^{n}}{\mathrm{~d} x}(x)-\frac{\mathrm{d} u_{i-1}^{n}}{\mathrm{~d} x}\left(x_{i-1}^{n}\right)+2 \frac{\phi^{\prime}(0)}{N}\left(x_{i-1}^{n}-x_{i}^{n}\right)
\end{aligned}
$$


Taking the integral over $\left(x_{i-1}^{n}, L\right]$, we obtain

$$
\begin{aligned}
\int_{x_{i-1}^{n}}^{L}\left|\frac{\mathrm{d} u_{i}^{n}}{\mathrm{~d} x}-\frac{\mathrm{d} u_{i-1}^{n}}{\mathrm{~d} x}\right| & \leq \int_{x_{i-1}^{n}}^{L}\left\{\frac{\mathrm{d} u_{i}^{n}}{\mathrm{~d} x}(x)-\frac{\mathrm{d} u_{i-1}^{n}}{\mathrm{~d} x}\left(x_{i-1}^{n}\right)+2 \phi^{\prime}(0)\left(x_{i-1}^{n}-x_{i}^{n}\right)\right\} \\
& =V_{i}^{n}-V_{i-1}^{n}-u_{i}^{n}\left(x_{i-1}^{n}\right)+u_{i-1}^{n}\left(x_{i-1}^{n}\right)+2 \frac{\phi^{\prime}(0)}{N}\left(x_{i-1}^{n}-x_{i}^{n}\right)\left(L-x_{i-1}^{n}\right) \\
& \leq V_{i}^{n}-V_{i-1}^{n}+2 \frac{\phi^{\prime}(0)}{N}\left(x_{i-1}^{n}-x_{i}^{n}\right)\left(L-x_{i-1}^{n}\right)
\end{aligned}
$$

using the fact that $u_{i-1}^{n} \leq u_{i}^{n}$.

As we combine the obtained estimates, we have

$$
\left\|\frac{\mathrm{d} u_{i}^{n}}{\mathrm{~d} x}-\frac{\mathrm{d} u_{i-1}^{n}}{\mathrm{~d} x}\right\|_{\mathbf{L}^{1}(0, L)} \leq V_{i}^{n}-V_{i-1}^{n}+2 \frac{\phi^{\prime}(0)}{N}\left[x_{i-1}^{n}-x_{i}^{n}\right]^{+}\left(L-x_{i-1}^{n}\right)+2 \frac{\phi^{\prime}(0)}{N}\left(\left[x_{i-1}^{n}-x_{i}^{n}\right]^{+}\right)^{2} .
$$

Suppose $V_{i-1}^{n}>V_{i-2}^{n}$.

By point IV of Corollary $1, x_{i}^{n} \leq x_{i-1}^{n}$. We have already seen that $\frac{\mathrm{d} u_{i}^{n}}{\mathrm{~d} x}=\frac{\mathrm{d} u_{i-1}^{n}}{\mathrm{~d} x}$ in $\left[0, x_{i}^{n}\right]$ and (in case $\left.x_{i}^{n}<x_{i-1}^{n}\right)$, for any $x$ in $\left(x_{i}^{n}, x_{i-1}^{n}\right]$,

$$
\left|\frac{\mathrm{d} u_{i}^{n}}{\mathrm{~d} x}(x)-\frac{\mathrm{d} u_{i-1}^{n}}{\mathrm{~d} x}(x)\right| \leq 2 \frac{\phi^{\prime}(0)}{N}\left(x_{i-1}^{n}-x_{i}^{n}\right) .
$$

For any $x$ in $\left(x_{i-1}^{n}, L\right]$, by the Euler-Lagrange equation, the monotonicity of $\phi_{-}^{\prime}$ and $\delta_{i-1}^{n} \leq \delta_{i}^{n}$, we have that

$$
N\left\{\frac{\mathrm{d}^{2} u_{i-1}^{n}}{\mathrm{~d} x^{2}}(x)-\frac{\mathrm{d}^{2} u_{i}^{n}}{\mathrm{~d} x^{2}}(x)\right\}=\phi_{-}^{\prime}\left(\delta_{i-1}^{n}(x)\right)-\phi_{-}^{\prime}\left(\delta_{i}^{n}(x)\right)
$$

hence,

$$
N\left\{\frac{\mathrm{d}^{2} u_{i-1}^{n}}{\mathrm{~d} x^{2}}(x)-\frac{\mathrm{d}^{2} u_{i}^{n}}{\mathrm{~d} x^{2}}(x)\right\} \geq 0
$$

Considering the integral on $\left(x_{i-1}^{n}, L\right]$,

$$
\begin{aligned}
& \int_{x_{i-1}^{n}}^{L}\left|\frac{\mathrm{d} u_{i}^{n}}{\mathrm{~d} x}-\frac{\mathrm{d} u_{i-1}^{n}}{\mathrm{~d} x}\right|=\int_{x_{i-1}^{n}}^{L}\left|\int_{x_{i-1}^{n}} \frac{\mathrm{d}^{2} u_{i}^{n}}{\mathrm{~d} x^{2}}-\frac{\mathrm{d}^{2} u_{i-1}^{n}}{\mathrm{~d} x^{2}}+\left(\frac{\mathrm{d} u_{i}^{n}}{\mathrm{~d} x}\left(x_{i-1}^{n}\right)-\frac{\mathrm{d} u_{i-1}^{n}}{\mathrm{~d} x}\left(x_{i-1}^{n}\right)\right)\right| \\
& \leq \int_{x_{i-1}^{n}}^{L}\left|\int_{x_{i-1}^{n}}\left\{\frac{\mathrm{d}^{2} u_{i}^{n}}{\mathrm{~d} x^{2}}-\frac{\mathrm{d}^{2} u_{i-1}^{n}}{\mathrm{~d} x^{2}}\right\}\right|+2 \frac{\phi^{\prime}(0)}{N}\left(x_{i-1}^{n}-x_{i}^{n}\right)\left(L-x_{i}^{n}\right) \\
& =\int_{x_{i-1}^{n}}^{L} \int_{x_{i-1}^{n}}\left\{\frac{\mathrm{d}^{2} u_{i-1}^{n}}{\mathrm{~d} x^{2}}-\frac{\mathrm{d}^{2} u_{i}^{n}}{\mathrm{~d} x^{2}}\right\}+2 \frac{\phi^{\prime}(0)}{N}\left(x_{i-1}^{n}-x_{i}^{n}\right)\left(L-x_{i}^{n}\right) \\
& =\int_{x_{i-1}^{n}}^{L}\left\{\frac{\mathrm{d} u_{i-1}^{n}}{\mathrm{~d} x}-\frac{\mathrm{d} u_{i}^{n}}{\mathrm{~d} x}-\frac{\mathrm{d} u_{i-1}^{n}}{\mathrm{~d} x}\left(x_{i-1}^{n}\right)+\frac{\mathrm{d} u_{i}^{n}}{\mathrm{~d} x}\left(x_{i-1}^{n}\right)\right\}+2 \frac{\phi^{\prime}(0)}{N}\left(x_{i-1}^{n}-x_{i}^{n}\right)\left(L-x_{i}^{n}\right) \\
& \leq V_{i-1}^{n}-V_{i}^{n}-u_{i-1}^{n}\left(x_{i-1}^{n}\right)+u_{i}^{n}\left(x_{i-1}^{n}\right)+4 \frac{\phi^{\prime}(0)}{N}\left(x_{i-1}^{n}-x_{i}^{n}\right)\left(L-x_{i}^{n}\right) .
\end{aligned}
$$


Since

$$
\begin{aligned}
\left|u_{i}^{n}\left(x_{i-1}^{n}\right)-u_{i-1}^{n}\left(x_{i-1}^{n}\right)\right|=\left|\int_{x_{i}^{n}}^{x_{i-1}^{n}} \frac{\mathrm{d} u_{i}^{n}}{\mathrm{~d} x}-\frac{\mathrm{d} u_{i-1}^{n}}{\mathrm{~d} x}\right| & \leq \int_{x_{i}^{n}}^{x_{i-1}^{n}}\left|\frac{\mathrm{d} u_{i}^{n}}{\mathrm{~d} x}-\frac{\mathrm{d} u_{i}^{n}}{\mathrm{~d} x}\left(x_{i}^{n}\right)+\frac{\mathrm{d} u_{i}^{n}}{\mathrm{~d} x}\left(x_{i}^{n}\right)-\frac{\mathrm{d} u_{i-1}^{n}}{\mathrm{~d} x}\right| \\
& \leq 2 \frac{\phi^{\prime}(0)}{N}\left(x_{i-1}^{n}-x_{i}^{n}\right)^{2},
\end{aligned}
$$

we get

$$
\int_{x_{i-1}^{n}}^{L}\left|\frac{\mathrm{d} u_{i}^{n}}{\mathrm{~d} x}-\frac{\mathrm{d} u_{i-1}^{n}}{\mathrm{~d} x}\right| \leq V_{i-1}^{n}-V_{i}^{n}+2 \frac{\phi^{\prime}(0)}{N}\left(x_{i-1}^{n}-x_{i}^{n}\right)^{2}+4 \frac{\phi^{\prime}(0)}{N}\left(x_{i-1}^{n}-x_{i}^{n}\right)\left(L-x_{i}^{n}\right) .
$$

We have obtained the estimate

$$
\left\|\frac{\mathrm{d} u_{i}^{n}}{\mathrm{~d} x}-\frac{\mathrm{d} u_{i-1}^{n}}{\mathrm{~d} x}\right\|_{\mathbf{L}^{1}(0, L)} \leq V_{i-1}^{n}-V_{i}^{n}+4 \frac{\phi^{\prime}(0)}{N}\left[x_{i-1}^{n}-x_{i}^{n}\right]^{+}\left(L-x_{i}^{n}\right)+2 \frac{\phi^{\prime}(0)}{N}\left(\left[x_{i-1}^{n}-x_{i}^{n}\right]^{+}\right)^{2} .
$$

The last subcase $V_{i-1}^{n}=V_{i-2}^{n}$ can be reconducted to one of the other two.

In fact, if $i=1$, then $V_{1}^{n}>V_{0}^{n}=V_{-1}^{n}=0$, and $u_{0}^{n}$ is the solution identically 0 and the proof is the same as the one in the first subcase.

For $i \geq 2$, either there exists a $j$ in $\{0, \ldots, i-3\}$ such that $V_{j}^{n} \neq V_{i-2}^{n}$, or $V_{i-2}^{n}=V_{i-3}^{n}=\cdots=0$. In this last case, $u_{i-1}^{n}$ is the solution identically 0 and the proof proceeds as in the first subcase. In case $V_{j}^{n} \neq V_{i-2}^{n}$, we take the greatest $j$ such that $V_{j}^{n} \neq V_{i-2}^{n}$. Since, as far as $V_{r}^{n}=V_{r+1}^{n}, u_{r}^{n}$ coincides with $u_{r+1}^{n}$ on the entire interval $[0, L]$, we can apply one of the previous subcases to the pair $V_{j}^{n}$ and $V_{i-1}^{n}$, where $V_{j}^{n}$ takes the role of $V_{i-2}^{n}$.

In any case, we can estimate the $\mathbf{L}^{1}(0, L)$ norm by

$$
\left\|\frac{\mathrm{d} u_{i}^{n}}{\mathrm{~d} x}-\frac{\mathrm{d} u_{i-1}^{n}}{\mathrm{~d} x}\right\|_{\mathbf{L}^{1}(0, L)} \leq\left|V_{i}^{n}-V_{i-1}^{n}\right|+6 \frac{\phi^{\prime}(0)}{N}\left[x_{i-1}^{n}-x_{i}^{n}\right]^{+} L .
$$

Notice that, by IV and V of Corollary $1, \sum_{i=1}^{p(n)}\left[x_{i-1}^{n}-x_{i}^{n}\right]^{+} \leq 2 h L$, where $h$ is the number of cycles of the displacement $V$, i.e. $h$ is such that $\cup_{r=1}^{h}\left(a_{r}, b_{r}\right)=\{t \in(0, T): \dot{V}$ is continuous at $\mathrm{t}, \dot{V}(t)>0\}$. The sought bound for the piecewise constant map $u^{n}$ is obtained summing over $i$ the inequality above:

$$
\begin{aligned}
\operatorname{Var}\left(\frac{\partial u^{n}}{\partial x} ;[0, T]\right)= & \sum_{i=1}^{p(n)}\left\|\frac{\partial u^{n}}{\partial x}\left(t_{i}^{n}\right)-\frac{\partial u^{n}}{\partial x}\left(t_{i-1}^{n}\right)\right\|_{\mathbf{L}^{1}(0, L)} \\
& \leq \sum_{i=1}^{p(n)}\left\{\left|V_{i}^{n}-V_{i-1}^{n}\right|+6 \frac{\phi^{\prime}(0)}{N}\left[x_{i-1}^{n}-x_{i}^{n}\right]^{+} L\right\} \\
& \leq\|\dot{V}\|_{\mathbf{L}^{1}(0, T)}+12 \frac{\phi^{\prime}(0)}{N} h L^{2}=: \mathrm{c}_{2} .
\end{aligned}
$$

That concludes the proof.

The estimates in Theorem 2 imply, by Helly's selection principle [1], that for any sequence of partitions $\left\{\mathcal{P}^{n}\right\}_{n}$ with step converging to 0 , there exists a subsequence $\left\{\mathcal{P}^{n_{k}}\right\}_{k}$ and a map

$$
u^{\prime} \text { in } \mathbf{L}^{\infty}\left((0, T) ; \mathbf{H}^{1}(0, L)\right) \cap \mathbf{B V}\left([0, T] ; \mathbf{L}^{1}(0, L)\right)
$$

such that, for any $t$ in $[0, T]$,

$$
\frac{\partial u^{n_{k}}}{\partial x}(t) \rightarrow u^{\prime}(t), \text { weakly in } \mathbf{H}^{1}(0, L)
$$


In particular, $u(t, x):=\int_{0}^{x} u^{\prime}(t)$ belongs to $\mathbf{H}^{2}(0, L)$, for any $t, \frac{\partial u}{\partial x}(t, x)=u^{\prime}(t, x)$, and

$$
\begin{aligned}
& u^{n_{k}}(t, x) \rightarrow u(t, x) \text { uniformly in } x \in[0, L], \\
& \frac{\partial u^{n_{k}}}{\partial x}(t) \rightarrow \frac{\partial u}{\partial x}(t) \text { strongly in } \mathbf{L}^{2}(0, L), \\
& \frac{\partial u^{n_{k}}}{\partial x}(t, L) \rightarrow \frac{\partial u}{\partial x}(t, L) .
\end{aligned}
$$

\section{The time Continuous evolution}

In this section we present our main result. We perform the limit of the time discrete evolutions as the time step goes to zero in order to construct the continuous formulation of the problem.

Before stating the result, we briefly introduce notation.

Let $v$ be a map from $[0, T]$ to $\mathbf{L}^{1}(0, L)$. We define the dissipation of $v$ in the interval of time $[s, t] \subset[0, T]$ by

$$
\operatorname{Diss}_{\phi}^{+}(v ;[s, t]):=\int_{0}^{L} \phi\left(\operatorname{Var}^{+}(v ;[s, t])\right),
$$

where $\operatorname{Var}^{+}(v(x) ;[s, t])$ is the positive variation of $v(x)$ in $[s, t]$, i.e. for any $f$ from $[0, T]$ to $\mathbb{R}$, the positive variation of $f$ in $[s, t]$ is defined by

$$
\operatorname{Var}^{+}(f ;[s, t]):=\operatorname{ess} \sup \left\{\sum_{k=1}^{l(n)}\left[f\left(\tau_{k}^{n}\right)-f\left(\tau_{k-1}^{n}\right)\right]^{+}: n \in \mathbb{N}, s=\tau_{0}^{n}<\tau_{1}^{n}<\cdots<\tau_{l(n)}^{n}=t\right\},
$$

where the essential supremum of an arbitrary family $\left\{f_{\alpha}\right\}_{\alpha \in A}$ of measurable functions $f_{\alpha}:(0, L) \rightarrow \mathbb{R}$ is the measurable function $f$ characterized by the following properties [13]:

- for every $\alpha, f_{\alpha} \leq f$ a.e. in $(0, L)$;

- if $g$ is a measurable function such that $f_{\alpha} \leq g$ a.e. in $(0, L)$, then $f \leq g$ a.e. in $(0, L)$.

The dissipation of $v$ is certainly finite whenever $v$ is a map of $\mathbf{B V}\left([0, T] ; \mathbf{L}^{1}(0, L)\right)$. In fact, arguing as in the proof of Proposition II-4-1 of [13], there exists a sequence of partitions $\left\{s=\tau_{0}^{n}<\tau_{1}^{n}<\cdots<\tau_{l(n)}^{n}=t\right\}$, increasing with respect to the inclusion, such that

$$
\operatorname{Var}^{+}(f ;[s, t])=\lim _{n \rightarrow \infty} \sum_{k=1}^{l(n)}\left[f\left(\tau_{k}^{n}\right)-f\left(\tau_{k-1}^{n}\right)\right]^{+} .
$$

By the monotone convergence theorem, using the concavity of $\phi$,

$\operatorname{Diss}_{\phi}^{+}(v ;[s, t])=\lim _{n \rightarrow \infty} \int_{0}^{L} \phi\left(\sum_{k=1}^{l(n)}\left[v\left(\tau_{k}^{n}\right)-v\left(\tau_{k-1}^{n}\right)\right]^{+}\right) \leq \phi^{\prime}(0) \lim _{n \rightarrow \infty} \sum_{k=1}^{l(n)} \int_{0}^{L}\left[v\left(\tau_{k}^{n}\right)-v\left(\tau_{k-1}^{n}\right)\right]^{+} \leq \phi^{\prime}(0) \operatorname{Var}(v ;[s, t])$.

Our main result is the following.

Theorem 3. Let $V:[0, T] \rightarrow[0, \infty)$ be a continuous displacement with piecewise continuous derivative on $[0, T]$ and $V(0)=0$. Assume that $V$ has a finite number of cycles, meaning that the open set $\{t \in(0, T)$ : $\dot{V}$ is continuous at $t, \dot{V}(t)>0\}$ has finitely many connected components.

Then, there exists an evolution map $u$ in $\mathbf{B V}\left([0, T] ; \mathbf{W}^{1,1}(0, L)\right) \cap \mathbf{L}^{\infty}\left([0, T] ; \mathbf{H}^{2}(0, L)\right)$, with $u(t, 0)=0$, $u(t, L)=V(t)$, such that $u(0)=0$ and, for any $t$ in $[0, T]$, 
I. $u(t)$ satisfies the inequality

$$
\frac{N}{2} \int_{0}^{L}\left|\frac{\partial u}{\partial x}(t)\right|^{2}+\operatorname{Diss}_{\phi}^{+}(u ;[0, t]) \leq \frac{N}{2} \int_{0}^{L}\left|\frac{\mathrm{d} v}{\mathrm{~d} x}\right|^{2}+\int_{0}^{L} \phi\left(\operatorname{Var}^{+}(u ;[0, t])+[v-u(t)]^{+}\right),
$$

for any $v$ in $\mathbf{W}^{1,2}(0, L), v(0)=0, v(L)=V(t)$

II. the total energy $\mathcal{E}(t):=\frac{N}{2} \int_{0}^{L}\left|\frac{\partial u}{\partial x}(t)\right|^{2}+\operatorname{Diss}_{\phi}^{+}(u ;[0, t])$ is an absolutely continuous function and is given by

$$
\mathcal{E}(t)=N \int_{0}^{t} \dot{V}(\tau) \frac{\partial u}{\partial x}(\tau, L) \mathrm{d} \tau
$$

Proof. Let $\cup_{r=1}^{h}\left\{a_{r}, b_{r}\right\} \subset[0, T]$ be the open set $\{t \in(0, T): \dot{V}$ is continuous at $t, \dot{V}(t)>0\}$.

Let $\left\{\mathcal{P}^{n}=\left\{0=t_{0}^{n}<t_{1}^{n}<\cdots<t_{p(n)}^{n}=T\right\}\right\}_{n}$ be a sequence of partitions of the interval $[0, T]$ with step converging to 0 such that $\cup_{r=1}^{h}\left\{a_{r}, b_{r}\right\} \subset \mathcal{P}^{n}$, for any $n$.

By passing to a subsequence, we can assume that $\left\{u^{n}\right\}_{n}$ enjoys the properties listed at the end of the previous section denoting $u \in \mathbf{B V}\left([0, T] ; \mathbf{W}^{1,1}(0, L)\right)$ the limit function.

Since $V(0)=0$, it follows that $u^{n}(0)=0$ for any $n$ and, since $u^{n}$ converges uniformly to $u, u(0)=0$.

We claim that, for any $t \in[0, T]$,

$$
\lim _{n \rightarrow \infty}\left\|\operatorname{Var}^{+}(u ;[0, t])-\operatorname{Var}^{+}\left(u^{n} ;[0, t]\right)\right\|_{\infty}=0
$$

This is due to the regularity of $\dot{V}$. Indeed, by Corollary $1, u^{n}(x)$ is non-decreasing in $\left(a_{r}, b_{r}\right)$, for any $x \in[0, L]$ and for any $r$. Let $r_{t}$ be such that $t \in\left(a_{r_{t}}, b_{r_{t}}\right)$. By the fact that $\mathcal{P}_{n}$ contains $\cup_{r=1}^{h}\left\{a_{r}, b_{r}\right\}$, we have

$$
\operatorname{Var}^{+}\left(u^{n}(x) ;[0, t]\right)=\sum_{r=1}^{r_{t}-1}\left\{u^{n}\left(b_{r}, x\right)-u^{n}\left(a_{r}, x\right)\right\}+u^{n}(t, x)-u^{n}\left(a_{r_{t}}, x\right)
$$

Since $u^{n}$ converges uniformly to $u$, it follows that $u(x)$ is non-decreasing in $\left(a_{r}, b_{r}\right)$, for any $x \in[0, L]$, and

$$
\lim _{n \rightarrow \infty} \operatorname{Var}^{+}\left(u^{n}(x) ;[0, t]\right)=\sum_{r=1}^{r_{t}-1}\left\{u\left(b_{r}, x\right)-u\left(a_{r}, x\right)\right\}+u(t, x)-u\left(a_{r_{t}}, x\right)=\operatorname{Var}^{+}(u(x) ;[0, t]),
$$

uniformly in $x \in[0, L]$.

Proof of I. Fix $t$ in $[0, T]$ and $v$ in $\mathbf{W}^{1,1}(0, L), v(0)=0, v(L)=V(t)$.

For any $n \in \mathbb{N}$, there exists $i=i(n)$ in $\mathcal{P}^{n}$, such that $t \in\left[t_{i}^{n}, t_{i+1}^{n}\right)$. Set $L_{n}:=L-\left|V(t)-V_{i}^{n}\right|$ and consider

$$
v_{i}^{n}(x):= \begin{cases}v(x), & x \in\left[0, L_{n}\right] \\ \left(x-L_{n}\right)\left(V_{i}^{n}-v\left(L_{n}\right)\right) /\left(L-L_{n}\right)+v\left(L_{n}\right), & x \in\left(L_{n}, L\right]\end{cases}
$$

$v_{i}^{n}$ belongs to $\mathbf{W}^{1,1}(0, L), v_{i}^{n}(0)=0, v_{i}^{n}(L)=V_{i}^{n}$ and $\left\|v_{i}^{n}-v\right\|_{\mathbf{H}^{1}(0, L)} \rightarrow 0$, as $n$ goes to $\infty$.

By the minimality of $u_{i}^{n}$, we have that

$$
\begin{aligned}
\frac{N}{2} \int_{0}^{L}\left|\frac{\mathrm{d} u_{i}^{n}}{\mathrm{~d} x}\right|^{2}+\int_{0}^{L} \phi\left(\delta_{i}^{n}\right) & \leq \frac{N}{2} \int_{0}^{L}\left|\frac{\mathrm{d} v_{i}^{n}}{\mathrm{~d} x}\right|^{2}+\int_{0}^{L} \phi\left(\delta_{i-1}^{n}+\left[v_{i}^{n}-u_{i}^{n}+u_{i}^{n}-u_{i-1}^{n}\right]^{+}\right) \\
& \leq \frac{N}{2} \int_{0}^{L}\left|\frac{\mathrm{d} v_{i}^{n}}{\mathrm{~d} x}\right|^{2}+\int_{0}^{L} \phi\left(\delta_{i}^{n}+\left[v_{i}^{n}-u_{i}^{n}\right]^{+}\right),
\end{aligned}
$$


and, by the fact that $\mathcal{P}_{n}$ contains $\cup_{r=1}^{h}\left\{a_{r}, b_{r}\right\}, \delta_{i}^{n}=\operatorname{Var}^{+}\left(u^{n} ;[0, t]\right)$.

Since $\frac{\mathrm{d} u^{n}}{\mathrm{~d} x}(t)$ converges strongly in $\mathbf{L}^{2}(0, L)$ to $\frac{\partial u}{\partial x}(t), u^{n}$ converges uniformly to $u$ and $\operatorname{Var}^{+}\left(u^{n} ;[0, t]\right)$ converges uniformly to $\operatorname{Var}^{+}(u ;[0, t])$ (see the observations at the end of the previous section), it follows that

$$
\frac{N}{2} \int_{0}^{L}\left|\frac{\partial u}{\partial x}(t)\right|^{2}+\operatorname{Diss}_{\phi}^{+}(u ;[0, t]) \leq \frac{N}{2} \int_{0}^{L}\left|\frac{\mathrm{d} v}{\mathrm{~d} x}\right|^{2}+\int_{0}^{L} \phi\left(\operatorname{Var}^{+}(u ;[0, t])+[v-u(t)]^{+}\right) .
$$

Proof of II. Fix $t$ in $[0, T]$. For any $n \in \mathbb{N}$, there exists $i=i(n)$ in $\mathcal{P}^{n}$, such that $t \in\left[t_{i}^{n}, t_{i+1}^{n}\right)$.

Set $\epsilon_{n}:=\max _{i \in\{1, \ldots, p(n)\}} \sqrt{\left|V_{i}^{n}-V_{i-1}^{n}\right|}$ and consider

$$
v_{i}^{n}(x):=u_{i-1}^{n}(x)+\left(V_{i}^{n}-V_{i-1}^{n}\right) \frac{\left[x-L+\epsilon_{n}\right]^{+}}{\epsilon_{n}} .
$$

One verifies that $v_{i}^{n}$ belongs to $\mathbf{W}^{1,1}(0, L), v_{i}^{n}(0)=0, v_{i}^{n}(L)=V_{i}^{n}$. By the minimality of $u_{i}^{n}$, we have that

$$
\begin{aligned}
& \frac{N}{2} \int_{0}^{L}\left|\frac{\mathrm{d} u_{i}^{n}}{\mathrm{~d} x}\right|^{2}+\int_{0}^{L} \phi\left(\delta_{i}^{n}\right) \\
& \leq \frac{N}{2} \int_{0}^{L}\left|\frac{\mathrm{d} u_{i-1}^{n}}{\mathrm{~d} x}\right|^{2}+\frac{N}{2} \int_{L-\epsilon_{n}}^{L} \frac{\left|V_{i}^{n}-V_{i-1}^{n}\right|^{2}}{\epsilon_{n}^{2}}+N \int_{L-\epsilon_{n}}^{L} \frac{\mathrm{d} u_{i-1}^{n}}{\mathrm{~d} x} \frac{V_{i}^{n}-V_{i-1}^{n}}{\epsilon_{n}} \\
&+\int_{0}^{L} \phi\left(\delta_{i-1}^{n}+\left[u_{i-1}^{n}+\left(V_{i}^{n}-V_{i-1}^{n}\right) \frac{\left[x-L+\epsilon_{n}\right]^{+}}{\epsilon_{n}}-u_{i-1}^{n}\right]^{+}\right) \\
& \leq \frac{N}{2} \int_{0}^{L}\left|\frac{\mathrm{d} u_{i-1}^{n}}{\mathrm{~d} x}\right|^{2}+\int_{0}^{L} \phi\left(\delta_{i-1}^{n}\right)+\frac{N}{2} \frac{\left|V_{i}^{n}-V_{i-1}^{n}\right|^{2}}{\epsilon_{n}}+N \frac{V_{i}^{n}-V_{i-1}^{n}}{\epsilon_{n}}\left\{u_{i-1}^{n}(L)-u_{i-1}^{n}\left(L-\epsilon_{n}\right)\right\} \\
&+\phi^{\prime}(0)\left[V_{i}^{n}-V_{i-1}^{n}\right]^{+} \frac{\epsilon_{n}}{2} .
\end{aligned}
$$

There exists a point $L_{k}$ in $\left(L-\epsilon_{n}, L\right)$, such that

$$
\left|\frac{u_{k-1}^{n}(L)-u_{k-1}^{n}\left(L-\epsilon_{n}\right)}{\epsilon_{n}}-\frac{\mathrm{d} u_{k-1}^{n}}{\mathrm{~d} x}(L)\right|=\left|\frac{\mathrm{d} u_{k-1}^{n}}{\mathrm{~d} x}\left(L_{k}\right)-\frac{\mathrm{d} u_{k-1}^{n}}{\mathrm{~d} x}(L)\right| \leq \epsilon_{n} \frac{\phi^{\prime}(0)}{N},
$$

where the bound is independent on $k$ (point I of Th. 2). Using this estimate, by iteration over $i$ as $i$ decreases to 0 , we obtain

$$
\begin{aligned}
& \frac{N}{2} \int_{0}^{L}\left|\frac{\mathrm{d} u_{i}^{n}}{\mathrm{~d} x}\right|^{2}+\int_{0}^{L} \phi\left(\delta_{i}^{n}\right) \leq \cdots \\
& \leq \frac{N}{2} \int_{0}^{L}\left|\frac{\mathrm{d} u_{0}^{n}}{\mathrm{~d} x}\right|^{2}+\int_{0}^{L} \phi\left(\delta_{0}^{n}\right)+\epsilon_{n} \frac{N}{2} \sum_{k=1}^{i}\left|V_{k}^{n}-V_{k-1}^{n}\right|+N \sum_{k=1}^{i}\left(V_{k}^{n}-V_{k-1}^{n}\right) \frac{u_{k-1}^{n}(L)-u_{k-1}^{n}\left(L-\epsilon_{n}\right)}{\epsilon_{n}} \\
& \quad+\epsilon_{n} \frac{\phi^{\prime}(0)}{2} \sum_{k=1}^{i}\left[V_{k}^{n}-V_{k-1}^{n}\right]^{+} \\
& \leq \frac{N}{2} \int_{0}^{L}\left|\frac{\mathrm{d} u_{0}^{n}}{\mathrm{~d} x}\right|^{2}+\int_{0}^{L} \phi\left(\delta_{0}^{n}\right)+N \sum_{k=1}^{i}\left\{V\left(t_{k}^{n}\right)-V\left(t_{k-1}^{n}\right)\right\} \frac{\partial u^{n}}{\partial x}\left(t_{k-1}^{n}, L\right)+\epsilon_{n} \frac{\|\dot{V}\|_{\mathbf{L}^{1}(0, T)}}{2}\left\{N+3 \phi^{\prime}(0)\right\} \\
& \quad=N \int_{0}^{t} \frac{\partial u^{n}}{\partial x}(\tau, L) \mathrm{d} V(\tau)+\epsilon_{n} \frac{\|\dot{V}\|_{\mathbf{L}^{1}(0, T)}}{2}\left\{N+3 \phi^{\prime}(0)\right\}
\end{aligned}
$$


The last equality above follows by the fact $\frac{\partial u^{n}}{\partial x}$ is a step function and the integral in the last member is in the sense of Riemann-Stieltjes [14].

Since $\delta_{i}^{n}=\operatorname{Var}^{+}\left(u^{n} ;[0, t]\right), \operatorname{Var}^{+}\left(u^{n} ;[0, t]\right)$ converges uniformly in $[0, L]$ to $\operatorname{Var}^{+}(u ;[0, t]), \frac{\partial u^{n}}{\partial x}(t)$ converges strongly in $\mathbf{L}^{2}(0, L)$ to $\frac{\partial u}{\partial x}(t)$ and $\frac{\partial u^{n}}{\partial x}(t, L)$ converges to $\frac{\partial u}{\partial x}(t, L)$ and is bounded uniformly with respect to $t$ (see Th. 2 and the observations at the end of the previous section), it follows that

$$
\begin{aligned}
\mathcal{E}(t) & =\lim _{n \rightarrow \infty}\left\{\frac{N}{2} \int_{0}^{L}\left|\frac{\partial u^{n}}{\partial x}(t)\right|^{2}+\operatorname{Diss}_{\phi}^{+}\left(u^{n} ;[0, t]\right)\right\}=\lim _{n \rightarrow \infty}\left\{\frac{N}{2} \int_{0}^{L}\left|\frac{\mathrm{d} u_{i}^{n}}{\mathrm{~d} x}\right|^{2}+\int_{0}^{L} \phi\left(\delta_{i}^{n}\right)\right\} \\
& \leq \lim _{n \rightarrow \infty}\left\{N \int_{0}^{t} \frac{\partial u^{n}}{\partial x}(\tau, L) \mathrm{d} V(\tau)\right\}=N \int_{0}^{t} \frac{\partial u}{\partial x}(\tau, L) \mathrm{d} V(\tau) .
\end{aligned}
$$

By the absolute continuity of $V$, we have that $\mathrm{d} V(\tau)=\dot{V}(\tau) \mathrm{d} \tau[14]$. Hence,

$$
\mathcal{E}(t) \leq N \int_{0}^{t} \frac{\partial u}{\partial x}(\tau, L) \dot{V}(\tau) \mathrm{d} \tau
$$

In order to prove the equality, we show also that the opposite inequality holds. The proof is similar to the previous case. The only difference is that we use the minimality of $u_{i-1}^{n}$, instead of $u_{i}^{n}$, an we slightly modify $u_{i}^{n}$ in order to compare them.

Consider $v_{i-1}^{n}(x):=u_{i}^{n}(x)+\left(V_{i-1}^{n}-V_{i}^{n}\right)\left[x-L+\epsilon_{n}\right]^{+} / \epsilon_{n}$. One verifies that $v_{i-1}^{n}$ belongs to $\mathbf{W}^{1,2}(0, L)$, $v_{i-1}^{n}(0)=0, v_{i-1}^{n}(L)=V_{i-1}^{n}$. By the minimality of $u_{i-1}^{n}$, we have that

$$
\begin{aligned}
& \frac{N}{2} \int_{0}^{L}\left|\frac{\mathrm{d} u_{i-1}^{n}}{\mathrm{~d} x}\right|^{2}+\int_{0}^{L} \phi\left(\delta_{i-1}^{n}\right) \\
& \leq \frac{N}{2} \int_{0}^{L}\left|\frac{\mathrm{d} u_{i}^{n}}{\mathrm{~d} x}\right|^{2}+\frac{N}{2} \int_{L-\epsilon_{n}}^{L} \frac{\left|V_{i-1}^{n}-V_{i}^{n}\right|^{2}}{\epsilon_{n}^{2}}+N \int_{L-\epsilon_{n}}^{L} \frac{\mathrm{d} u_{i}^{n}}{\mathrm{~d} x} \frac{V_{i-1}^{n}-V_{i}^{n}}{\epsilon_{n}} \\
&+\int_{0}^{L} \phi\left(\delta_{i-2}^{n}+\left[u_{i}^{n}+\left(V_{i-1}^{n}-V_{i}^{n}\right) \frac{\left[x-L+\epsilon_{n}\right]^{+}}{\epsilon_{n}}-u_{i-2}^{n}\right]^{+}\right) \\
& \leq \frac{N}{2} \int_{0}^{L}\left|\frac{\mathrm{d} u_{i}^{n}}{\mathrm{~d} x}\right|^{2}+\int_{0}^{L} \phi\left(\delta_{i}^{n}\right)+\frac{N}{2} \frac{\left|V_{i-1}^{n}-V_{i}^{n}\right|^{2}}{\epsilon_{n}}+N \frac{V_{i-1}^{n}-V_{i}^{n}}{\epsilon_{n}}\left\{u_{i}^{n}(L)-u_{i}^{n}\left(L-\epsilon_{n}\right)\right\} \\
&+\phi^{\prime}(0)\left[V_{i-1}^{n}-V_{i}^{n}\right]^{+} \frac{\epsilon_{n}}{2} .
\end{aligned}
$$

By iteration over $i$, as $i$ decreases to 0 , we obtain

$$
\begin{aligned}
0= & \frac{N}{2} \int_{0}^{L}\left|\frac{\mathrm{d} u_{0}^{n}}{\mathrm{~d} x}\right|^{2}+\int_{0}^{L} \phi\left(\delta_{0}^{n}\right) \leq \cdots \\
\leq & \frac{N}{2} \int_{0}^{L}\left|\frac{\mathrm{d} u_{i}^{n}}{\mathrm{~d} x}\right|^{2}+\int_{0}^{L} \phi\left(\delta_{i}^{n}\right)+\epsilon_{n} \frac{N}{2} \sum_{k=1}^{i}\left|V_{k}^{n}-V_{k-1}^{n}\right|+N \sum_{k=1}^{i}\left(V_{k-1}^{n}-V_{k}^{n}\right) \frac{u_{k-1}^{n}(L)-u_{k-1}^{n}\left(L-\epsilon_{n}\right)}{\epsilon_{n}} \\
& +\epsilon_{n} \frac{\phi^{\prime}(0)}{2} \sum_{k=1}^{i}\left[V_{k-1}^{n}-V_{k}^{n}\right]^{+} \\
\leq & \frac{N}{2} \int_{0}^{L}\left|\frac{\mathrm{d} u_{i}^{n}}{\mathrm{~d} x}\right|^{2}+\int_{0}^{L} \phi\left(\delta_{i}^{n}\right)-N \sum_{k=1}^{i}\left\{V\left(t_{k}^{n}\right)-V\left(t_{k-1}^{n}\right)\right\} \frac{\partial u^{n}}{\partial x}\left(t_{k-1}^{n}, L\right)+\epsilon_{n} \frac{\|\dot{V}\|_{\mathbf{L}^{1}(0, T)}}{2}\left\{N+3 \phi^{\prime}(0)\right\} \\
= & \frac{N}{2} \int_{0}^{L}\left|\frac{\mathrm{d} u_{i}^{n}}{\mathrm{~d} x}\right|^{2}+\int_{0}^{L} \phi\left(\delta_{i}^{n}\right)-N \int_{0}^{t} \frac{\partial u^{n}}{\partial x}(\tau, L) \mathrm{d} V(\tau)+\epsilon_{n} \frac{\|\dot{V}\|_{\mathbf{L}^{1}(0, T)}}{2}\left\{N+3 \phi^{\prime}(0)\right\} .
\end{aligned}
$$


It follows that

$$
\begin{aligned}
\mathcal{E}(t) & =\lim _{n \rightarrow \infty}\left\{\frac{N}{2} \int_{0}^{L}\left|\frac{\partial u^{n}}{\partial x}(t)\right|^{2}+\operatorname{Diss}_{\phi}^{+}\left(u^{n} ;[0, t]\right)\right\}=\lim _{n \rightarrow \infty}\left\{\frac{N}{2} \int_{0}^{L}\left|\frac{\mathrm{d} u_{i}^{n}}{\mathrm{~d} x}\right|^{2}+\int_{0}^{L} \phi\left(\delta_{i}^{n}\right)\right\} \\
& \geq \lim _{n \rightarrow \infty}\left\{N \int_{0}^{t} \frac{\partial u^{n}}{\partial x}(\tau, L) \mathrm{d} V(\tau)\right\}=N \int_{0}^{t} \frac{\partial u}{\partial x}(\tau, L) \mathrm{d} V(\tau)
\end{aligned}
$$

hence,

That concludes the proof.

$$
\mathcal{E}(t) \geq N \int_{0}^{t} \frac{\partial u}{\partial x}(\tau, L) \dot{V}(\tau) \mathrm{d} \tau .
$$

Acknowledgements. The author wish to thank Gregoire Allaire for his support, Gilles Francfort and Jean-Jacques Marigo for having introduced him to this subject, and anonymous referee for several useful suggestions.

The research has been supported by the Marie Curie MULTIMAT "Multi-scale, Modelling and Characterisation for Phase Transformations in Advanced Materials" Research Training Network under the contract number MRTN-CT-2004505226 .

\section{REFERENCES}

[1] V. Barbu and T. Precupanu, Convexity and optimization in Banach spaces. D. Reidel Publishing Co., Dordrecht (1986).

[2] B. Dacorogna, Direct methods in the calculus of variations. Springer-Verlag, Berlin (1989).

[3] G. Dal Maso and R. Toader, A model for the quasi-static evolution of brittle fractures: existence and approximation results. Arch. Rational Mech. Anal. 162 (2002) 102-135.

[4] G. Dal Maso, G.A. Francfort and R. Toader, Quasi-static crack growth in finite elasticity. Arch. Rational Mech. Anal. 176 (2005) 165-225.

[5] G.A. Francfort and A. Garroni, A variational view of partial brittle damage evolution. Arch. Rational Mech. Anal. 182 (2006) $125-152$.

[6] G.A. Francfort and C. Larsen, Existence and convergence for quasi-static evolution in brittle fractures. Comm. Pure Applied Math. 56 (2003) 1495-1500.

[7] G.A. Francfort and J.-J. Marigo, Revisiting brittle fracture as an energy minimization problem. J. Mech. Phys. Solids 46 (1998) 1319-1342.

[8] G.A. Francfort and A. Mielke, Existence results for a class of rate-independent material models with nonconvex elastic energies. J. reine angew. Mathematik 595 (2006) 55-91.

[9] A. Friedman, Variational principles and free-boundary problems. Wiley-Interscience (1982).

[10] A. Griffith, The phenomena of rupture and flow in solids. Philos. Trans. Roy. Soc. London Ser. A 221 (1920) $163-198$.

[11] A. Jaubert and J.-J. Marigo, L'approche variationnelle de la fatigue: des premiers résultats. C. R. Mecanique 333 (2005) $550-556$.

[12] D. Mumford and J. Shah, Optimal approximations by piecewise smooth functions and associated variational problems. Comm. Pure Appl. Math. 42 (1989) 577-685.

[13] J. Neveu, Bases mathématiques du calcul des probabilités. Masson Cie, Paris (1970).

[14] R. Wheeden and A. Zygmund, Measure and integral. Marcel Dekker (1977). 